$$
S-56460-D
$$

ThE ASTROPHYSICAL JOURNAL, 478:745-765, 1997 April 1

(C) 1997. The American Astronomical Society. All rights reserved. Printed in U.S.A.

$$
\text { NAS5-2655 }
$$

NASA-CR -204643

$03035 \%$

\title{
EVALUATING POSSIBLE HEATING MECHANISMS USING THE TRANSITION REGION LINE PROFILES OF LATE-TYPE STARS ${ }^{1}$
}

\author{
BRIAN E. WOOD AND JEFFrey L. LINSKY \\ JILA, University of Colorado, Boulder, CO 80309-0440; woodb@marmot.colorado.edu,jlinsky@jila.colorado.edu \\ AND \\ ThOMAS R. AYReS \\ Center for Astrophysics and Space Astronomy, University of Colorado, Boulder, CO 80309-0391; \\ ayres@vulcan.colorado.edu \\ Received 1996 July 1 ; accepted 1996 October 23
}

\begin{abstract}
Our analysis of high-resolution Goddard High-Resolution Spectrograph (GHRS) spectra of late-type stars shows that the $\mathrm{Si}$ IV and $\mathrm{C}$ IV lines formed near $10^{5} \mathrm{~K}$ can be decomposed into the sum of two Gaussians, a broad component and a narrow component. We find that the flux contribution of the broad components is correlated with both the C IV and X-ray surface fluxes. For main-sequence stars, the widths of the narrow components suggest subsonic nonthermal velocities, and there appears to be a tight correlation between these nonthermal velocities and stellar surface gravity $\left(\xi_{\mathrm{NC}} \propto g^{-0.68 \pm 0.07}\right)$. For evolved stars with lower surface gravities, the nonthermal velocities suggested by the narrow components are at or just above the sound speed. Nonthermal velocities computed from the widths of the broad components are always highly supersonic. We propose that the broad components are diagnostics for microflare heating. Turbulent dissipation and Alfvén waves are both viable candidates for the narrow component heating mechanism.

A solar analog for the broad components might be the "explosive events" detected by the HighResolution Telescope and Spectrograph (HRTS) experiment. The broad component we observe for the Si IV 21394 line of $\alpha$ Cen A, a star that is nearly identical to the Sun, has a FWHM of $109 \pm 10 \mathrm{~km} \mathrm{~s}^{-1}$ and is blueshifted by $9 \pm 3 \mathrm{~km} \mathrm{~s}^{-1}$ relative to the narrow component. Both of these properties are consistent with the properties of the solar explosive events. However, the $\alpha$ Cen A broad component accounts for $25 \% \pm 4 \%$ of the total Si IV line flux, while solar explosive events are currently thought to account for no more than $5 \%$ of the Sun's total transition region emission. This discrepancy must be resolved before the connection between broad components and explosive events can be positively established.

In addition to our analysis of the Si IV and $\mathrm{C}$ IV lines of many stars, we also provide a more thorough analysis of all of the available GHRS data for $\alpha$ Cen A (G2 V) and $\alpha$ Cen B (K1 V). We find that the transition region lines of both stars have redshifts almost identical to those observed on the Sun: showing an increase with line formation temperature up to about $\log T=5.2$ and then a rapid decrease. Using the $\mathrm{O} \mathrm{IV}]$ lines as density diagnostics, we compute electron densities of $\log n_{e}=9.65 \pm 0.20$ and $\log n_{e}=9.50 \pm 0.30$ for $\alpha$ Cen A and $\alpha$ Cen B, respectively.

Subject headings: line: profiles — stars: activity - stars: chromospheres — stars: coronae stars: late-type - ultraviolet: stars
\end{abstract}

\section{INTRODUCTION}

Edlén's (1941) identification of highly ionized species in the solar corona and the subsequent detection of solar and stellar X-rays and ultraviolet emission lines demonstrated that the outer atmospheres of late-type stars are heated to temperatures greatly in excess of the photospheric surface temperatures. These heated regions are called chromospheres (for the $T \leq 2 \times 10^{4} \mathrm{~K}$ plasma), transition regions (for the $2 \times 10^{4} \mathrm{~K} \leq T \leq 10^{6} \mathrm{~K}$ plasma), and coronae (for the $T \geq 10^{6} \mathrm{~K}$ plasma). The existence of the hot gas requires nonradiative, or "mechanical," heating processes. Despite five decades of effort, there has been only limited success in deducing the nature of these heating processes. Proposals include the dissipation of acoustic waves via

\footnotetext{
${ }^{1}$ Based on observations with the NASA/ESA Hubble Space Telescope, obtained at the Space Telescope Science Institute, which is operated by the Association of Universities for Research in Astronomy Inc., under NASA Contract NAS5-26555.
}

shocks (proposed as early as the 1940s), turbulent or shock wave dissipation of various modes of MHD waves, Joule heating by electric currents, quasi-steady magnetic field reconnection, and rapid field reconnection either as lowenergy transient events (microflaring) or high-energy events (flaring). On the Sun, the transition region and coronal emission arises predominantly in regions dominated by magnetic fields, which suggests the existence of a magnetic heating mechanism. For a comprehensive review of possible heating mechanisms, see Narain \& Ulmschneider (1990, 1996) and the papers from the Heidelberg conference "Mechanisms of Chromospheric and Coronal Heating" (Ulmschneider, Priest, \& Rosner 1991).

The main difficulty in identifying which mechanism(s) heats the chromosphere or corona of a specific late-type star or a class of such stars is that one observes only the gross effects of the heating process, with no spatial resolution and (usually) poor time resolution. Stellar X-ray luminosities and the broadband spectral energy distributions observed with the Einstein, ROSAT, and ASCA satellites, and line 
TABLE 1

Stellar Parameters

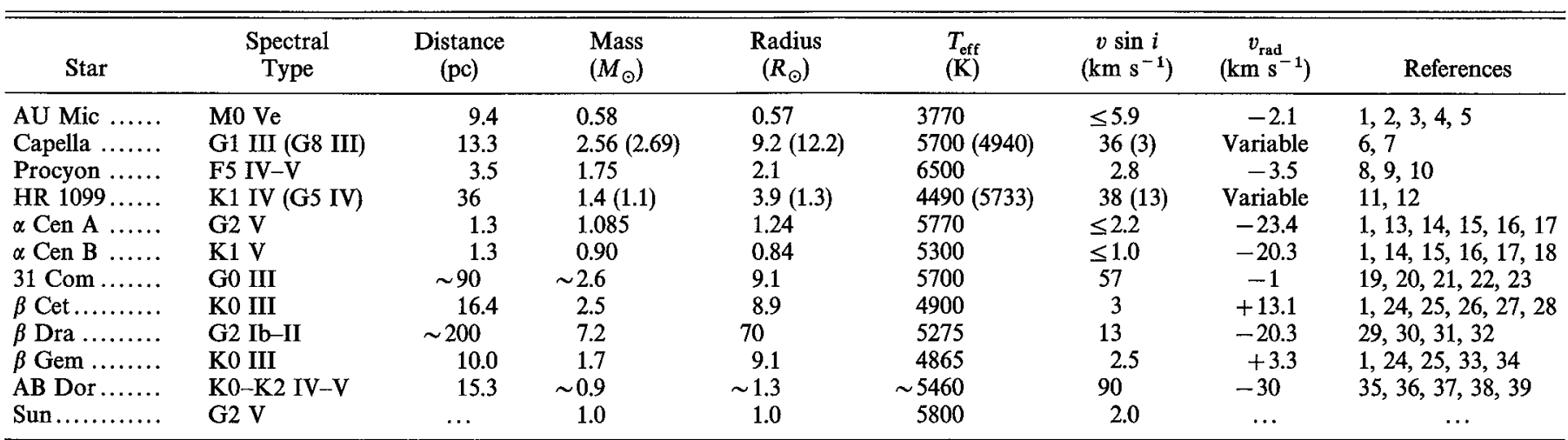

ReFerences.-(1) Gliese \& Jahreiss 1991; (2) Pettersen 1980; (3) Bopp \& Fekel 1977; (4) Henry \& McCarthy 1993; (5) Linsky \& Wood 1994; (6) Hummel et al. 1994; (7) Strassmeier \& Fekel 1990; (8) Irwin et al. 1992; (9) Gray 1982a; (10) Steffen 1985; (11) Fekel 1983; (12) Vogt \& Penrod 1983; (13) Hallam, Altner, \& Endal 1991;(14) Heintz 1982;(15) Lydon, Fox, \& Sofia 1993; (16) Demarque, Guenther, \& van Altena 1986; (17) Noels et al. 1991; (18) Char et al. 1993; (19) Ayres et al. 1995; (20) Strassmeier et al. 1990; (21) Hirshfeld, Sinnott, \& Ochsenbein 1991; (22) Oranje, Zwaan, \& Middlekoop 1982; (23) Flower 1977; (24) Gray 1982b; (25) Astronomical Almanac 1996; (26) Eriksson, Linsky, \& Simon 1983; (27) Iben 1967; (28) Bell \& Gustafsson 1978; (29) Brown et al. 1984; (30) Hoffleit \& Jaschek 1982; (31) Beavers \& Eitter 1986; (32) Luck 1982; (33) Blackwell et al. 1990; (34) Drake \& Smith 1991; (35) Rucinski 1985; (36) Guirado et al. 1996; (37) Vilhu et al. 1993; (38) Vilhu et al. 1996; (39) Hempelmann et al. 1995.

fluxes observed with $E U V E$, provide information on the corona's response to heating, but no clear signatures of the nature of the heating mechanism(s) itself. Similarly, the fluxes of ultraviolet emission lines observed with $I U E$ and Hubble Space Telescope (HST) provide information on the emission measure distribution in the chromosphere and transition region, which again is simply a response to the heating. What is needed are observational discriminators that can identify the different heating mechanisms.

The intensity of the observed emission and its correlation with stellar parameters provides an initial crude discriminator between different types of heating processes. For example, Schrijver (1987) and others have noted that the radiative losses per unit area of a star (the stellar surface flux) in important emission lines formed in chromospheres and transition regions have minimum values that depend upon $B-V$ color (or $T_{\text {eff }}$ ) and upon gravity. This so called "basal flux," observed from old slowly rotating stars and also seen in the centers of solar supergranule cells away from strong magnetic fields, is generally thought to be the result of heating by shocks from simple acoustic waves generated in the stellar convective zone (see, e.g., Ulmschneider 1990). Such heating must always be present and is the logical explanation for the weak background emission level seen in the low-activity stars.

Enhanced X-ray and UV-line surface fluxes characterize "active stars," a generic term that includes pre-mainsequence stars, young main-sequence stars, $\mathrm{dMe}$ and other flare stars, and rapidly rotating binaries (e.g., RS CVn, Algol, and W UMa systems). The active stars have X-ray and UV-line surface fluxes up to $10^{4}$ times larger than the basal flux stars. For some diagnostics like X-rays, Mg II, and $C$ IV, there appears to be an empirical upper limit to the surface flux that is now called "saturated heating" (Vilhu 1987). The excess heating above the basal rate is empirically correlated with stellar rotation and is generally thought to be magnetic in character, but until now it has been unclear whether the heating is due to the dissipation by turbulence or shock waves in a magnetic field (e.g., MHD waves; Stein 1981 ) or to the rapid annihilation of magnetic fields to produce accelerated particles and subsequent heating (see, e.g., Parker 1988), a process often called "microflaring." In this paper, we describe a simple diagnostic signature that might allow the relative importance of these two types of magnetic heating processes to be estimated empirically.

Linsky \& Wood (1994) discovered that HST/GHRS profiles of the Si IV and C IV emission lines of the dM0e flare star AU Mic, which are formed at a temperature of about $10^{5} \mathrm{~K}$ in the transition region, contain broad wings extending out to at least $\pm 200 \mathrm{~km} \mathrm{~s}^{-1}$. They found that the AU Mic profiles could be fitted with two Gaussians, a narrow component and a broad component. In Wood's (1996) reanalysis of these data, the widths of the two components were found to be FWHM $=31 \pm 3 \mathrm{~km} \mathrm{~s}^{-1}$ and FWHM $=162 \pm 11 \mathrm{~km} \mathrm{~s}^{-1}$, which suggests most probable nonthermal velocities of $\xi=15.4 \pm 1.5 \mathrm{~km} \mathrm{~s}^{-1}$ and $\xi=97 \pm 9 \mathrm{~km} \mathrm{~s}^{-1}$, respectively. The nonthermal velocity of the narrow component is somewhat less than nonthermal velocities observed in the solar transition region $\left(\xi_{\odot} \approx 26\right.$ $\mathrm{km} \mathrm{s}^{-1}$; Dere \& Mason 1993), but it is the nonthermal velocity of the broad component that is strikingly different. Linsky \& Wood (1994) proposed that microflaring in the transition region of $\mathrm{AU}$ Mic is responsible for the broad components, which implies that microflaring is an important source of atmospheric heating for AU Mic.

Linsky et al. (1995) and Wood (1996) also detected broad components in the transition region lines of Capella and showed that the broad wings are not present in chromospheric lines such as the $\mathrm{Mg}_{\mathrm{II}} h$ and $k$ lines and the $\mathrm{O}_{\mathrm{I}}$ $\lambda 1300$ triplet. For the G1 III star, which provides about $91 \%$ of the flux in the transition region lines, they found that the broad components are responsible for about $41 \%$ of the total Si IV and C Iv flux. Broad components were also detected in the spectra of the cooler star (K1 IV) of the RS CVn system HR 1099 (Wood et al. 1996). The chromospheric Mg II $h$ and $k$ lines of HR 1099 also have broad wings, which suggests that for HR 1099, the broad component phenomenon might extend into the chromosphere, although the very high opacity of the $\mathrm{Mg}$ II lines makes this interpretation uncertain. The $\mathrm{C} \mathrm{IV}$ broad components of the K1 IV star, which produces essentially all of the line flux of HR 1099 , contribute about $66 \%$ of the total line flux. In 
contrast, Wood et al. (1996) found no broad components ( $\leq 20 \%$ of the total line flux) in the C IV and Si rv profiles of the relatively inactive star Procyon (F5 IV-V); although the $\mathrm{N} v$ line, which is formed at higher temperatures, does appear to have a broad component that accounts for about $42 \%$ of the $\mathrm{N} v$ flux.

In this paper, we use GHRS observations of seven additional stars to investigate stellar transition region line profiles further, specifically to explore the nature of the broad wing emission seen in the $\mathrm{C}$ IV and $\mathrm{Si}$ IV lines of $\mathrm{AU}$ Mic, Capella, and HR 1099. The full sample of stars is summarized in Table 1. Two sets of stellar parameters are listed for Capella and HR 1099, as both are close, unresolved binary systems. The parameters given in parentheses are for the star that is only a minor contributor to the UV emission lines. Vilhu et al. (1996) used two-Gaussian fits to model the $\mathrm{C}$ IV lines of the Pleiades age star AB Dor (K0-K2 IV-V), similar to our analyses of the C IV lines of AU Mic, Capella, and HR 1099, so those results also are considered here. We present a complete analysis of the available GHRS observations of $\alpha$ Centauri A (G2 V) and $\alpha$ Centauri B (K1 V). We also discuss GHRS observations of $\beta$ Ceti (K0 III), 31 Comae (G0 III), $\beta$ Geminorum (K0 III), and $\beta$ Draconis (G2 $\mathrm{Ib}-\mathrm{II})$. However, because the GHRS data sets for these four stars are considerably larger than the very limited $\alpha$ Cen $\mathrm{A}+\mathrm{B}$ data set, we confine our attention to only the C IV and $\mathrm{Si}$ Iv lines. More thorough analyses of the $\beta \mathrm{Cet}, 31 \mathrm{Com}, \beta$ $\mathrm{Gem}$, and $\beta$ Dra data will be presented in future papers.

\section{GHRS OBSERVATIONS}

The GHRS instrument and its capabilities prior to the installation of the COSTAR corrective optics in 1993 December are described in detail by Brandt et al. (1994) and Heap et al. (1995). COSTAR significantly improved the spectral resolution of observations made through the large science aperture (LSA). Table 2 lists the GHRS spectra analyzed here, most of which are post-COSTAR, LSA observations.

The fundamental data are moderate-resolution observations of the C IV $\lambda 1550$ and Si rV $\lambda 1400$ spectral regions, except for $\alpha$ Cen A and $\alpha$ Cen B for which we lack the C IV spectra. For $\beta$ Dra, we have C IV spectra obtained in three different years. Echelle spectra of $\alpha$ Cen A and $\alpha$ Cen B containing the Lyman- $\alpha, \mathrm{O}$ v] $\lambda 1218$, and $\mathrm{Mg}$ II $h$ and $k$ lines are listed also in Table 2. These observations already have been presented by Linsky \& Wood (1996) in an analysis of interstellar absorption features. We refer the reader to that paper for a description of the data reduction performed on those observations. The procedures used for the moderate-resolution data are discussed briefly below; they are described in more detail in Wood et al. (1996), Wood (1996), Linsky et al. (1993), and references therein.

The Pt-Ne calibration lamp was observed before each of the observations listed in Table 2 (except for the $\alpha$ Cen A G140M observation - see $\$ 3.1$ ), and these spectra were used to calibrate the wavelengths of the science spectra. All of the observations were subdivided into two or more readouts (see Table 2). Breaking up the observations in this fashion allows us to correct for line shifts induced during the course of the observations by changes in Earth's magnetic field and/or instrumental thermal effects. The wavelengths of the individual readouts are corrected by cross-correlating them against the first readout, which is taken nearest in time to the wavelength calibration image. We also searched the individual readouts for flux variations that might indicate flaring. No such variations were detected, however.

The $\beta$ Cet, 31 Com, and $\beta$ Gem spectra were obtained using the FP-SPLIT mode, in which the spectrum is shifted on the diode array between readouts several times during the observation. This technique allows one to compensate for sensitivity variations in the photocathode that is positioned in front of the diode array (Heap et al. 1995).

\section{THE GHRS SPECTRA OF $\alpha$ CENTAURI A AND $\alpha$ CENTAURI B}

\subsection{Data Analysis}

The nearby $\alpha$ Cen star system has been extensively studied in the past. In the ultraviolet, both $\alpha$ Cen A and B have been popular targets for the IUE. One of the more extensive analyses of IUE observations of $\alpha$ Cen A and B is that of Jordan et al. (1987), who used the IUE data to create simple one-dimensional models of the atmospheric structure of the two stars. Ayres et al. (1995) and Hallam, Altner, \& Endal (1991) have investigated the time variation of the UV line fluxes of $\alpha$ Cen A and B. Of particular interest is the substantial long-term variability noted by Ayres et al. (1995) for $\alpha$ Cen B, which may indicate a magnetic cycle similar to the $11 \mathrm{yr}$ solar cycle and the activity cycles observed on

TABLE 2

GHRS OBSERVATIONS

\begin{tabular}{|c|c|c|c|c|c|c|c|c|}
\hline Star & Grating & Aperture & $\begin{array}{c}\text { Spectral Range } \\
(\AA)\end{array}$ & $\begin{array}{l}\text { Spectral Resolution } \\
\left(\mathrm{km} \mathrm{s}^{-1}\right)\end{array}$ & Date & $\begin{array}{l}\text { Start Time } \\
\text { (UT) }\end{array}$ & $\begin{array}{l}\text { Exposure Time } \\
\text { (s) }\end{array}$ & $\begin{array}{c}\text { Number of } \\
\text { Readouts }\end{array}$ \\
\hline \multirow{3}{*}{$\alpha \operatorname{Cen} \mathrm{A} \ldots \ldots$} & G140M & LSA & $1390-1417$ & 11 & 1995 May 1 & $14: 38$ & 485 & 2 \\
\hline & Ech-A & SSA & $1212-1219$ & 3.5 & 1995 May 1 & $15: 02$ & 2786 & 12 \\
\hline & Ech-B & SSA & $2792-2807$ & 3.5 & 1995 May 1 & $17: 40$ & 485 & 1 \\
\hline \multirow[t]{3}{*}{$\alpha \operatorname{Cen} B \ldots \ldots$} & Ech-B & SSA & $2792-2807$ & 3.5 & 1995 May 5 & $10: 25$ & 700 & 1 \\
\hline & G140M & LSA & $1390-1417$ & 11 & 1995 May 5 & $11: 44$ & 700 & 2 \\
\hline & Ech-A & SSA & $1212-1219$ & 3.5 & 1995 May 5 & $12: 04$ & 3231 & 12 \\
\hline \multirow{2}{*}{$\beta$ Cet $\ldots \ldots \ldots$} & G160M & LSA & $1385-1421$ & 15 & 1994 Jun 2 & $22: 58$ & 2585 & 8 \\
\hline & G160M & LSA & $1537-1573$ & 15 & 1994 Jun 3 & $00: 36$ & 1293 & 4 \\
\hline \multirow{2}{*}{31 Com ....... } & G160M & LSA & $1385-1421$ & 15 & 1994 Nov 7 & $22: 39$ & 3878 & 12 \\
\hline & G160M & LSA & $1537-1573$ & 15 & 1994 Nov 8 & $01: 30$ & 1293 & 4 \\
\hline \multirow[t]{4}{*}{$\beta$ Dra $\ldots . . .}$. & G160M & LSA & $1532-1568$ & 20 & 1992 Apr 23 & $20: 55$ & 889 & 3 \\
\hline & G160M & LSA & $1532-1568$ & 20 & 1993 Feb 20 & $21: 15$ & 889 & 3 \\
\hline & G160M & LSA & $1383-1419$ & 15 & 1995 Apr 29 & $22: 23$ & 1777 & 6 \\
\hline & G160M & LSA & $1532-1568$ & 15 & 1995 Apr 30 & $00: 09$ & 889 & 3 \\
\hline \multirow[t]{2}{*}{$\beta \mathrm{Gem}$} & G160M & LSA & $1532-1568$ & 15 & 1995 Sep 20 & $02: 08$ & 1293 & 4 \\
\hline & G160M & LSA & $1384-1420$ & 15 & 1995 Sep 20 & $03: 29$ & 2047 & 4 \\
\hline
\end{tabular}




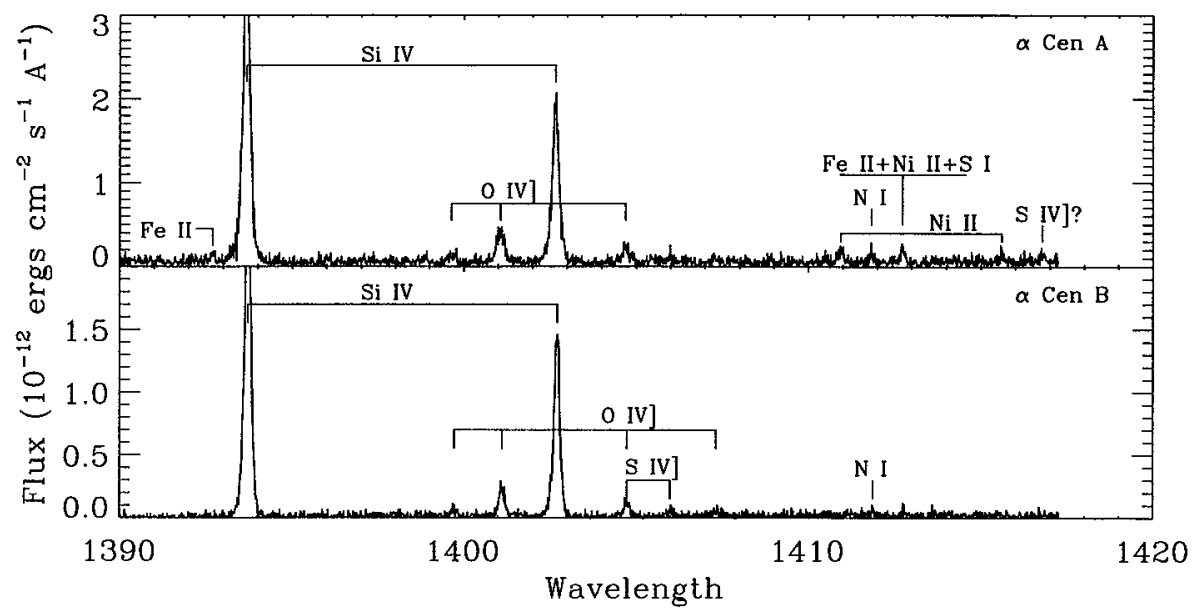

FIG. 1. GHRS spectra of the Si IV spectral regions of $\alpha$ Cen A and $\alpha$ Cen B, with line identifications

many other solar-like stars using optical observations of the $\mathrm{Ca}$ II $\mathrm{H}$ and $\mathrm{K}$ lines (Baliunas et al. 1995).

Our GHRS $\alpha$ Cen A and B data sets unfortunately are very small, although the quality of the data is much superior to the IUE data. The G140M spectra of the 1390$1417 \AA$ spectral regions of $\alpha$ Cen A and $\alpha$ Cen B are shown in Figure 1. Besides the Si IV and O IV] lines, which were the main objectives for these observations, we also have identified several weak chromospheric lines using the solar spectral atlas of Sandlin et al. (1986). We measured the velocities, widths (FWHM), and fluxes of these lines using single-Gaussian fits. The best fits were determined by $\chi^{2}$ minimization (see, e.g., Bevington \& Robinson 1992). The resulting parameters are listed in Tables 3 and 4. The $1 \sigma$ random errors in the tables were estimated using Monte Carlo techniques. For all of the fits in this paper, we correct for instrumental broadening by convolving each proposed fit with the instrumental profile, which we assume is a Gaussian with a FWHM of 4.4 pixels (Gilliland 1994).

Besides the lines identified in the 1390-1417 $\AA$ spectral region, we also list the parameters of the $\mathrm{H} \mathrm{I}, \mathrm{O} \mathrm{v}]$, and $\mathrm{Mg}$ II lines observed in echelle spectra analyzed by Linsky \& Wood (1996). The self-reversed H I Lyman- $\alpha$ and Mg II $h$ and $k$ lines have high opacities and thus are not close to Gaussian in shape. We estimated velocities for these lines according to the centroid of the line wings, and we estimated fluxes using corrections for interstellar absorption from Linsky \& Wood (1996). The O v] $\lambda 1218$ lines are shown explicitly in Figure 2.

Laboratory wavelengths in Tables 3 and 4 are from Kelly (1987) and Sandlin et al. (1986), except for the $O$ v] $\lambda 1218$ wavelength, which is from Brown (1980). Based on the orbital parameters of Heintz (1982), we compute radial velocities of -23.4 and $-20.3 \mathrm{~km} \mathrm{~s}^{-1}$ for $\alpha$ Cen A and $\alpha$

TABLE 3

Line Profile Parameters for $\alpha$ Cen A

\begin{tabular}{|c|c|c|c|c|c|c|}
\hline Ion & $\begin{array}{l}\lambda_{\text {rest }} \\
(\AA)\end{array}$ & $\begin{array}{c}\lambda_{\text {meas }} \\
(\AA)\end{array}$ & $\begin{array}{c}v^{a} \\
\left(\mathrm{~km} \mathrm{~s}^{-1}\right)\end{array}$ & $\begin{array}{c}f \\
\left(10^{-13}\right)\end{array}$ & $\begin{array}{c}\text { FWHM } \\
\left(\mathrm{km} \mathrm{s}^{-1}\right)\end{array}$ & $\chi_{v}^{2}$ \\
\hline \multicolumn{7}{|c|}{ Single-Gaussian Fits } \\
\hline 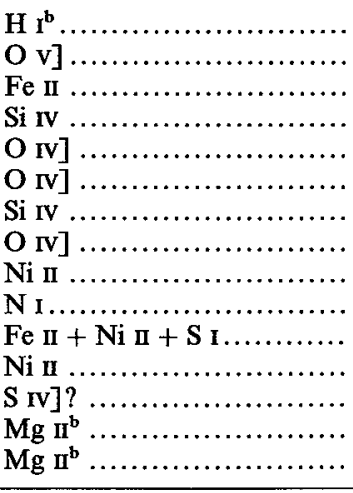 & $\begin{array}{l}1215.670 \\
1218.344 \\
1392.817 \\
1393.755 \\
1399.774 \\
1401.156 \\
1402.770 \\
1404.806 \\
1411.071 \\
1411.949 \\
\ldots \\
1415.728 \\
1416.930 \\
2795.528 \\
2802.705 \\
\end{array}$ & $\begin{array}{l}1215.571 \\
1218.259 \\
1392.707 \\
1393.673 \\
1399.657 \\
1401.082 \\
1402.681 \\
1404.707 \\
1410.955 \\
1411.848 \\
1412.745 \\
1415.613 \\
1416.799 \\
2795.310 \\
2802.486 \\
\end{array}$ & $\begin{array}{r}-1 \pm 1 \\
2 \pm 4 \\
0 \pm 3 \\
6 \pm 1 \\
-2 \pm 5 \\
8 \pm 2 \\
4 \pm 1 \\
2 \pm 3 \\
-1 \pm 3 \\
2 \pm 3 \\
-1 \pm 3 \\
-4 \pm 5 \\
0 \pm 1 \\
0 \pm 1 \\
\end{array}$ & $\begin{array}{l}1200 \pm 200 \\
3.1 \pm 0.4 \\
0.12 \pm 0.03 \\
9.5 \pm 0.1 \\
0.21 \pm 0.04 \\
0.98 \pm 0.06 \\
4.7 \pm 0.1 \\
0.42 \pm 0.05 \\
0.24 \pm 0.04 \\
0.15 \pm 0.03 \\
0.25 \pm 0.03 \\
0.14 \pm 0.03 \\
0.16 \pm 0.03 \\
2250 \pm 80 \\
1730 \pm 80 \\
\end{array}$ & $\begin{array}{l}65 \pm 9 \\
18 \pm 6 \\
59 \pm 1 \\
53 \pm 12 \\
55 \pm 4 \\
56 \pm 1 \\
54 \pm 8 \\
38 \pm 7 \\
27 \pm 7 \\
26 \pm 5 \\
30 \pm 7 \\
35 \pm 8 \\
\cdots \\
\cdots \\
\end{array}$ & $\begin{array}{l}0.577 \\
0.957 \\
2.198 \\
1.109 \\
0.858 \\
1.179 \\
1.036 \\
0.992 \\
0.992 \\
0.992 \\
1.040 \\
1.040 \\
\cdots \\
\cdots \\
\end{array}$ \\
\hline \multicolumn{7}{|c|}{ Two-Gaussian Fits } \\
\hline Si IV . & $\begin{array}{l}1393.755 \\
1393.755\end{array}$ & $\begin{array}{l}1393.678 \\
1393.639\end{array}$ & $\begin{array}{r}7 \pm 1 \\
-2 \pm 3\end{array}$ & $\begin{array}{l}7.4 \pm 0.5 \\
2.5 \pm 0.4\end{array}$ & $\begin{array}{c}47 \pm 2 \\
109 \pm 10\end{array}$ & $\begin{array}{l}1.252 \\
1.252\end{array}$ \\
\hline
\end{tabular}

a The line velocity relative to the star, assuming a stellar radial velocity of $-23.4 \mathrm{~km} \mathrm{~s}^{-1}$.

b The fluxes and velocities of the $\mathrm{Ly} \alpha$ and $\mathrm{Mg}$ II lines were measured directly rather than by a Gaussian fit. 
TABLE 4

Line Profite Parameters for $\alpha$ Cen B

\begin{tabular}{|c|c|c|c|c|c|c|}
\hline Ion & $\begin{array}{l}\lambda_{\text {rest }} \\
(\AA)\end{array}$ & $\begin{array}{l}\lambda_{\text {meas }} \\
(\AA)\end{array}$ & $\begin{array}{c}v^{\mathrm{a}} \\
\left(\mathrm{km} \mathrm{s}^{-1}\right)\end{array}$ & $\begin{array}{c}f \\
\left(10^{-13}\right)\end{array}$ & $\begin{array}{c}\text { FWHM } \\
\left(\mathrm{km} \mathrm{s}^{-1}\right)\end{array}$ & $\chi_{v}^{2}$ \\
\hline \multicolumn{7}{|c|}{ Single-Gaussian Fits } \\
\hline 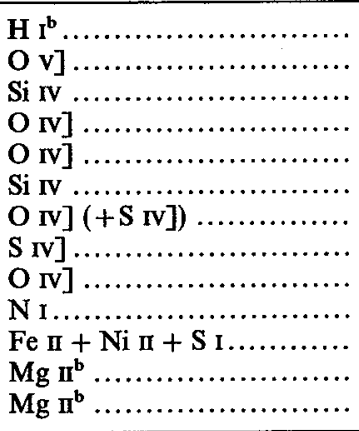 & $\begin{array}{c}1215.670 \\
1218.344 \\
1393.755 \\
1399.774 \\
1401.156 \\
1402.770 \\
1404.806 \\
1406.060 \\
1407.386 \\
1411.949 \\
\ldots \ldots \\
2795.528 \\
2802.705\end{array}$ & $\begin{array}{l}1215.588 \\
1218.275 \\
1393.710 \\
1399.719 \\
1401.121 \\
1402.716 \\
1404.738 \\
1405.990 \\
1407.323 \\
1411.884 \\
1412.764 \\
2795.339 \\
2802.515\end{array}$ & $\begin{array}{r}0 \pm 1 \\
3 \pm 1 \\
11 \pm 1 \\
9 \pm 4 \\
13 \pm 1 \\
9 \pm 1 \\
6 \pm 2 \\
5 \pm 6 \\
7 \pm 6 \\
6 \pm 5 \\
\cdots \\
0 \pm 1 \\
0 \pm 1\end{array}$ & $\begin{array}{l}1800 \pm 400 \\
1.8 \pm 0.1 \\
6.4 \pm 0.1 \\
0.10 \pm 0.02 \\
0.47 \pm 0.03 \\
3.09 \pm 0.07 \\
0.21 \pm 0.02 \\
0.09 \pm 0.02 \\
0.07 \pm 0.02 \\
0.04 \pm 0.02 \\
0.05 \pm 0.02 \\
1640 \pm 60 \\
1210 \pm 60\end{array}$ & $\begin{array}{l}40 \pm 3 \\
50 \pm 1 \\
40 \pm 9 \\
42 \pm 3 \\
48 \pm 1 \\
38 \pm 6 \\
45 \pm 11 \\
43 \pm 11 \\
14 \pm 9 \\
17 \pm 8 \\
\quad \cdots \\
\quad \cdots \\
\end{array}$ & $\begin{array}{c}0.813 \\
2.253 \\
0.721 \\
0.792 \\
1.505 \\
0.709 \\
0.974 \\
1.066 \\
0.884 \\
0.884 \\
\cdots \\
\cdots\end{array}$ \\
\hline \multicolumn{7}{|c|}{ Two-Gaussian Fits } \\
\hline $\begin{array}{l}\text { Si IV ..... } \\
\text { Si IV ...... }\end{array}$ & $\begin{array}{l}1393.755 \\
1393.755 \\
1402.770 \\
1402.770\end{array}$ & $\begin{array}{l}1393.711 \\
1393.686 \\
1402.722 \\
1402.697\end{array}$ & $\begin{array}{r}11 \pm 1 \\
5 \pm 2 \\
11 \pm 1 \\
5 \pm 2\end{array}$ & $\begin{array}{l}4.7 \pm 0.3 \\
1.8 \pm 0.3 \\
2.3 \pm 0.2 \\
0.9 \pm 0.2\end{array}$ & $\begin{array}{l}39 \pm 2 \\
86 \pm 6 \\
39 \pm 2 \\
86 \pm 6\end{array}$ & $\begin{array}{l}0.953 \\
0.953 \\
0.953 \\
0.953\end{array}$ \\
\hline
\end{tabular}

a The line velocity relative to the star, assuming a stellar radial velocity of $-20.3 \mathrm{~km} \mathrm{~s}^{-1}$.

- The fluxes and velocities of the Ly $\alpha$ and $\mathrm{Mg}$ II lines were measured directly rather than by a Gaussian fit.

Cen $B$ at the time of the observations. Tables 3 and 4 list the line velocities in the stellar rest frame.

The wavelengths of the $\alpha$ Cen B spectra should be very accurate, because these observations were obtained immediately following wavelength calibration images. The Lyman- $\alpha$ and Mg II $h$ and $k$ line wings appear to be well

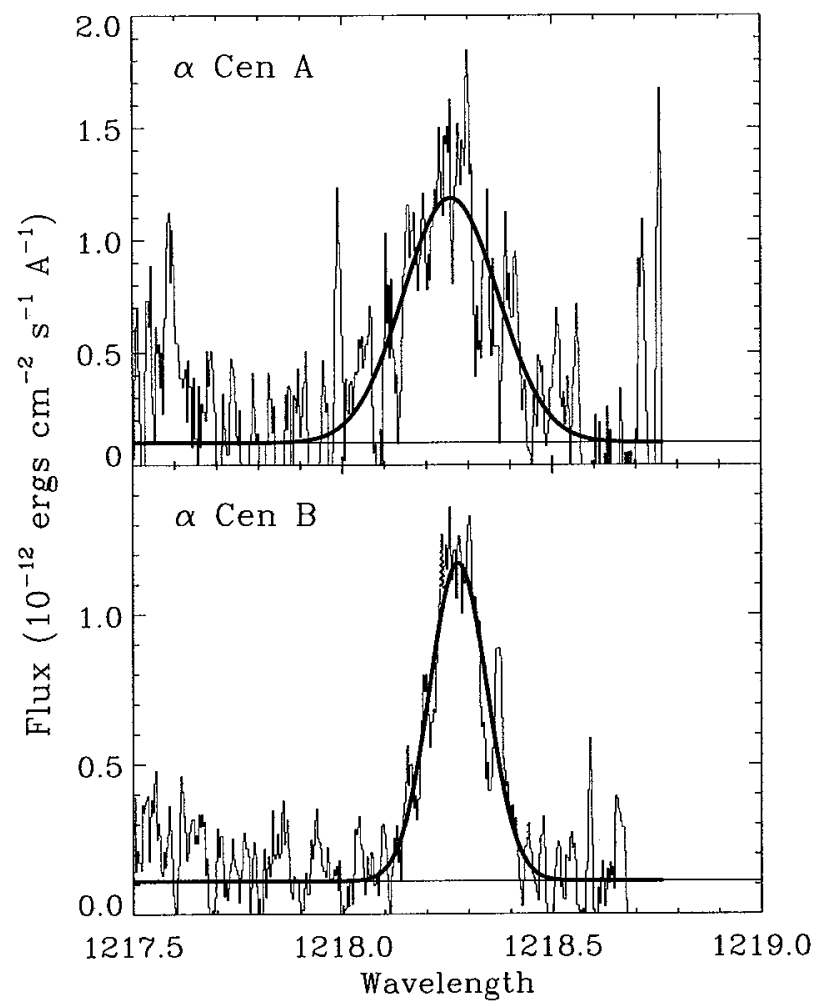

FIG. 2.-Single-Gaussian fits to the $O$ v] $\lambda 1218$ lines of $\alpha$ Cen A and B observed with the Ech-A grating of the GHRS. The data have been smoothed slightly for the sake of clarity. The parameters of these fits are listed in Tables 3 and 4. centered on the rest frame of the star, which confirms the accuracy of the wavelength calibrations. The wavelength scale of the G140M spectrum of $\alpha$ Cen A is more uncertain, however, because the wavelength calibration image attempted prior to the observation was not completed successfully. We were therefore forced to use the default wavelength calibration for this spectrum, although we did do an offset correction as described by Soderblom, Sherbert, \& Hulbert (1993).

Initially, we found that the $\mathrm{Fe}$ II, $\mathrm{Ni}$ II, and $\mathrm{N}$ I lines of $\alpha$ Cen A appeared to be blueshifted relative to the star by an average of $3 \mathrm{~km} \mathrm{~s}^{-1}$. The blueshifts are unlikely to be genuine, because on the Sun and solar-like stars, weak chromospheric lines such as these tend to be at the stellar rest velocity or slightly redshifted (see, e.g., Wood et al. 1996; Achour et al. 1995; Hassler, Rottman, \& Orrall 1991). Therefore, we attribute the apparent blueshifts to an inaccurate wavelength calibration, and we compensate for them by increasing the wavelengths of the G140M spectrum by $0.014 \AA$ (i.e., $3 \mathrm{~km} \mathrm{~s}^{-1}$ ). The velocities in Table 3 are the corrected values. We note that the $\mathrm{H}$ I and $\mathrm{Mg}$ II lines of $\alpha$ Cen A appear to be centered on the stellar radial velocity, which suggests that there is no problem with the wavelength calibration of the echelle data.

The last column of Tables 3 and 4 lists the $\chi_{v}^{2}$ values for the fits. In this paper, a fit will be considered acceptable if it is a $2 \sigma$ fit, which means that the probability that random errors cannot account for the discrepancies between the fit and the data is $\leq 95.4 \%$. (If the probability is $\leq 68.3 \%$, it is a $1 \sigma$ fit, and so on.) The exact value of $\chi_{v}^{2}$ that separates the acceptable fits (i.e., $2 \sigma$ fits) from unacceptable fits depends on the number of degrees of freedom, $v$, which for our purposes is basically the number of data points in the spectral region being fitted (see Bevington \& Robinson 1992). This value will generally be about $\chi_{v}^{2}=1.25 \pm 0.10$.

Inspection of the $\chi_{v}^{2}$ values in Tables 3 and 4 reveals that the single-Gaussian fits to the stronger Si rv line of $\alpha$ Cen A and both Si Iv lines of $\alpha$ Cen B are not acceptable. There- 
fore, following the example of Linsky \& Wood (1994), Linsky et al. (1995), and Wood et al. (1996), we fitted these lines with two Gaussians. In order to constrain the fits to the Si IV lines of $\alpha$ Cen B, the two lines were fitted simultaneously, forcing the narrow and broad components of the two lines to have the same velocities and widths. For both the broad and narrow components, we also forced the Si IV $\lambda 1394 / \lambda 1403$ flux ratio to be the optically thin value of 2 . The two-Gaussian fits are shown in Figure 3, and the fit parameters are given in Tables 3 and 4 . These results will be discussed further in $\S 5$.

\subsection{Line Velocities}

Skylab and more recent rocket experiments have clearly demonstrated that the transition region lines of the Sun are redshifted relative to the solar photosphere and chromosphere (see, e.g., Doschek, Feldman, \& Bohlin 1976; Hassler et al. 1991; Brekke 1993). The IUE has discovered similar line redshifts on many other stars, including $\alpha$ Cen A and B (Ayres, Jensen, \& Engvold 1988). On the Sun, the observed redshifts are correlated with line formation temperature,

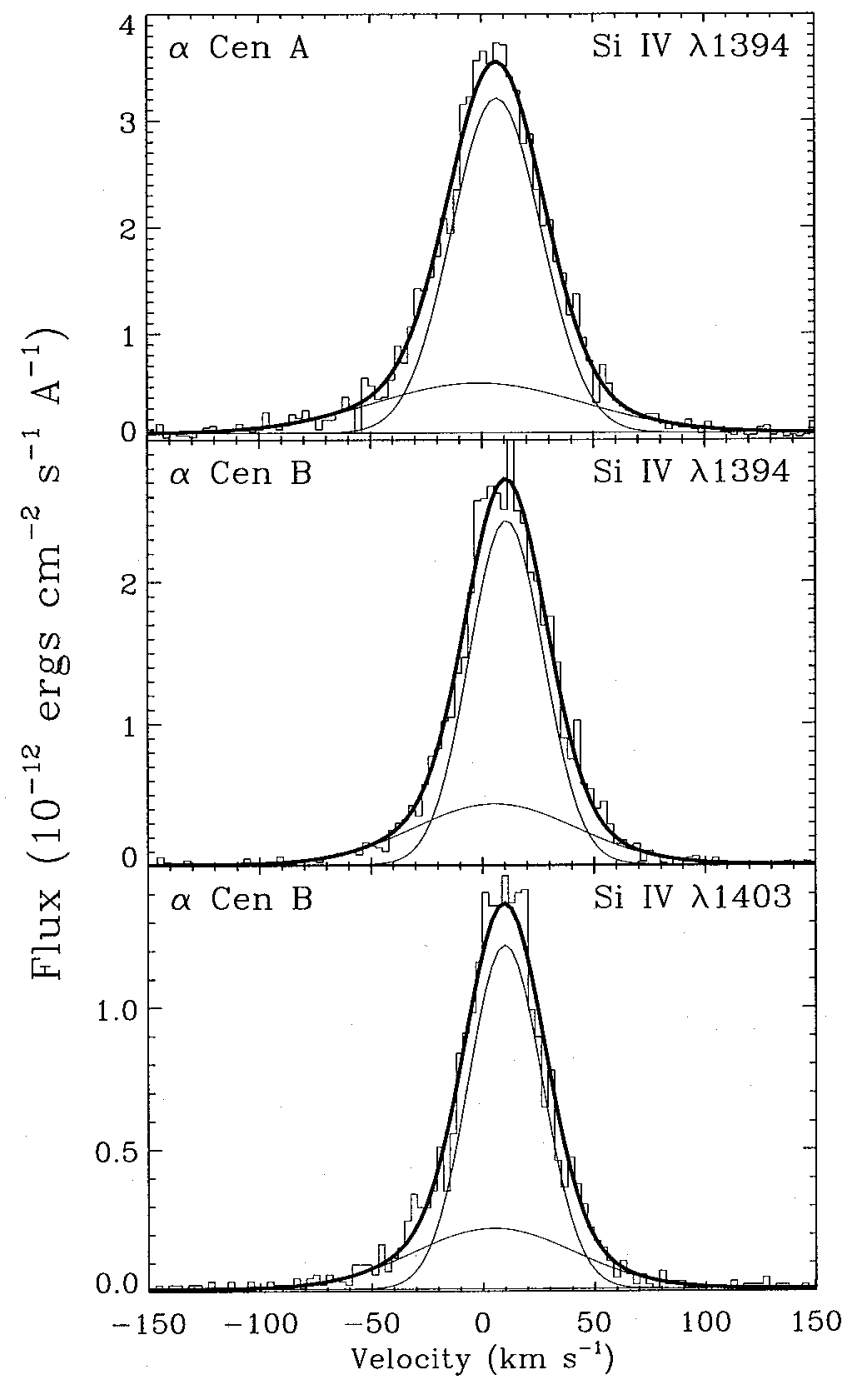

FIG. 3.-Two-Gaussian fits to the Si rv lines of $\alpha$ Cen A and B. The data are shown in histogram form. For each fit, the individual Gaussian components are displayed as thin solid lines, and the thick solid line is the convolution of the sum of the components with the instrumental profile. The parameters of these fits are listed in Tables 3 and 4. with a steady increase of line redshift up to about $10 \mathrm{~km} \mathrm{~s}^{-1}$ at $\log T=5.2$. The behavior of the redshifts above $\log T=5.2$ is somewhat controversial. Based on the $\mathrm{N} \mathrm{v}$ $\lambda \lambda 1239,1243$ and $O \mathrm{v}] \lambda 1218$ lines, which are formed at about $\log T=5.3$, there is evidence that the redshifts decrease dramatically above $\log T=5.2$. However, Brekke (1993) pointed out that this apparent decrease in redshift could be spurious due to uncertainties in the rest wavelengths of the aforementioned $\mathrm{N} \mathrm{v}$ and $\mathrm{O} \mathrm{v}$ ] lines. Indeed, the solar $\mathrm{O} v \lambda 1371$ velocity is not consistent with the $\mathrm{N} v$ and $\mathrm{O} v$ ] velocities, and suggests at most only a small decrease in redshift for temperatures above $\log T=5.2$. This issue is an important one, because the origin of the transition region line redshifts remains controversial, and the dependence of the redshifts on line formation temperature provides a potentially useful test of theoretical models that seek to explain the redshifts.

The $\alpha$ Cen A and B line lists in Tables 3 and 4 may be short, but the data have unprecedented quality, and the lines span a broad range of temperature-from $\log T=4.1$ for the $\mathrm{Mg}$ II lines to $\log T=5.3$ for the $\mathrm{O}$ v] $\lambda 1218$ line. Thus, we can at least crudely determine the variation of line redshifts with temperature. Figure 4 depicts the line redshifts of $\alpha$ Cen A and B as a function of formation temperature. We plot the velocity of only the strongest $\mathrm{O}$ IV] line, and we restrict consideration to the measurements with errors less than $\pm 4 \mathrm{~km} \mathrm{~s}^{-1}$. The line formation temperatures were estimated from the centroids of the contribution functions (see, e.g., Brown et al. 1984; Jordan et al. 1987) and the ionization equilibrium calculations of Arnaud \& Rothenflug (1985). The line redshifts of the Sun and Procyon are also shown in Figure 4, based on data presented by Achour et al. (1995) and Wood et al. (1996).

Below temperatures of about $\log T=5.2$, the line redshifts of the Sun, $\alpha$ Cen A, $\alpha$ Cen B, and Procyon are all very similar, although the $\mathrm{Si}$ IV and $\mathrm{O}$ IV] redshifts of $\alpha$ Cen B appear to be roughly $4 \mathrm{~km} \mathrm{~s}^{-1}$ larger than for the other three stars. Above $\log T=5.2$, the $\mathrm{O} v] \lambda 1218$ lines of both $\alpha$ Cen A and B suggest drop-offs in redshift similar to that suggested by the solar data, when the same $\mathrm{O} v$ ] rest wavelength is assumed. This is not the case for Procyon, however. The rest wavelengths of the $\mathrm{N} \mathrm{v}$ and $\mathrm{O} v$ ] lines, which suggest drop-offs in the line redshifts above $\log T=5.2$ for the Sun, $\alpha$ Cen A, and $\alpha$ Cen B, are 1238.821 $\AA$ and 1218.344 $\AA$, respectively. Using these same rest wavelengths, we find no drop-off in line redshifts for Procyon, as previously noted by Wood et al. (1996). This provides strong evidence that the decrease in line redshift seen for the Sun, $\alpha$ Cen A, and $\alpha$ Cen $B$ is real and not due to inaccuracies in the rest wavelengths. The fact that the redshift decrease is seen for the Sun, $\alpha$ Cen A, and $\alpha$ Cen B, but is not seen for Procyon, a hotter and somewhat more active star, might represent an important insight into the origin of the effect.

Achour et al. (1995) list a velocity of $9.0 \mathrm{~km} \mathrm{~s}^{-1}$ for the solar O v $\lambda 1371$ line. We did not plot this velocity in Figure 4 because this line velocity is inconsistent with the $\mathrm{N} \mathrm{V}$ $\lambda 1239$ and $\mathrm{O} v] \lambda 1218$ velocities. If the rest wavelengths of $\mathrm{N} \mathrm{v}$ and $\mathrm{O} v]$ are accurate, as we have argued, then the rest wavelength of the $\mathrm{O} \vee \lambda 1371$ line $(1371.292 \AA)$ is suspect.

\subsection{Transition Region Densities}

The $\mathrm{O}$ Iv] lines seen in Figure 1 are very useful as density diagnostics (Cook et al. 1995). All four of the O Iv] lines are 


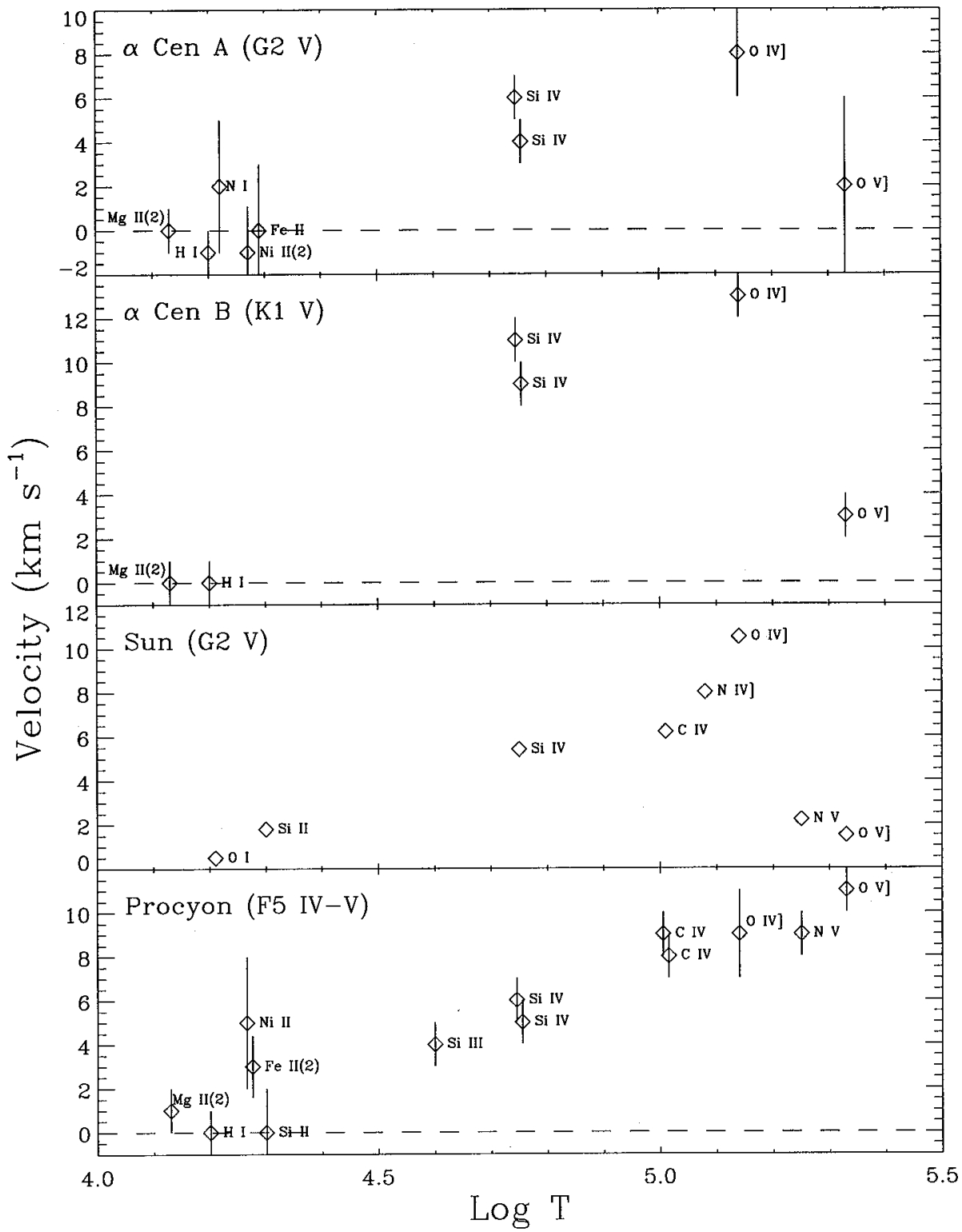

Fig. 4.- Line velocities of $\alpha$ Cen A, $\alpha$ Cen B, the Sun, and Procyon plotted as a function of line formation temperature. The data for the Sun and Procyon are from Achour et al. (1995) and Wood et al. (1996), respectively. The atomic species associated with each data point is indicated in the figure. In a number of instances, the velocities of two lines of the same atomic species are averaged, and only one data point is used to represent the lines in the figure. In these instances, the number of lines represented by the data point is indicated by a number in parentheses.

detected in the $\alpha$ Cen B spectrum, while only three are clearly visible for $\alpha$ Cen A. For the latter, there appears to be a flux excess near $1407 \AA$ that probably is the O IV] 1407.4 $\AA$ line, but we cannot make an accurate measurement of this feature owing to the uneven continuum and possible line blends. However, we do have a flux measurement for the O IV] $1399.8 \AA$ line, which should have the same flux as the $\lambda 1407.4$ line for all densities (Cook et al. 1995). In the midst of the O IV] multiplet falls the S IV] $1406.1 \AA$ line, which is clearly visible in the $\alpha$ Cen B spectrum. There is a feature at $1406 \AA$ in the $\alpha$ Cen A spectrum that probably is the S IV] line, but as was the case for O IV] 21407.4, the uneven continuum and possible line blends prevent us from measuring the flux of the feature. Another complication is a S IV] line that is blended with $\mathrm{O}$ IV] 21404.8. However, Cook et al. (1995) find that for relatively low densities, $S$ IV] $\lambda 1404.8$ should have only $21 \%$ of the flux of S IV] $\lambda 1406.1$, which means that the O IV] $1404.8 \AA$ line accounts for at least $90 \%$ of the flux of the $1405 \AA$ feature in the spectra of both $\alpha$ Cen A and B. Nevertheless, for $\alpha$ Cen B we do reduce the O IV] $\lambda 1404.8$ flux reported in Table 4 by $10 \%$ (based on the measured S IV] $\lambda 1406.1$ flux) before proceeding with the analysis.

There are three useful density-sensitive line ratios that can be computed from the $O$ IV] lines: $R_{1}=f_{1400} / f_{1401}$, $R_{2}=f_{1400} / f_{1405}$, and $R_{3}=f_{1405} / f_{1401}$. For $\alpha$ Cen $\mathrm{B}$, the average flux of the $\lambda 1399.8$ and $\lambda 1407.4$ lines is used for $f_{1400}$, since these two lines should have the same flux for all densities. From these three flux ratios and the $\mathrm{O}$ IV model of Cook et al. (1995), we compute three values for the electron density of each star. The flux ratios and corresponding densities are listed in Table 5 . Note that for $\alpha$ Cen $\mathrm{B}, R_{1}$ and $R_{2}$ provide only upper limits for the density. The densities suggested by the three ratios are reasonably self-consistent, with $R_{3}$ providing the most accurate density measurement for both stars. Collectively, the results in Table 5 suggest 
TABLE 5

Electron Densities From O IV] Line RATIOS

\begin{tabular}{ccr}
\hline Star & Line Ratio $^{\mathrm{a}}$ & \multicolumn{1}{c}{$\log n_{e}$} \\
\hline$\alpha$ Cen A $\ldots \ldots \ldots \ldots \ldots$. & $R_{1}=0.21 \pm 0.04$ & $10.14_{-1.10}^{+0.44}$ \\
& $R_{2}=0.55 \pm 0.13$ & $9.79_{-0.31}^{+0.23}$ \\
& $R_{3}=0.39 \pm 0.06$ & $9.62_{-0.23}^{+0.22}$ \\
$\alpha$ Cen B $\ldots \ldots \ldots \ldots \ldots$ & $R_{1}=0.18 \pm 0.05$ & $9.59_{-\infty}^{+0.80}$ \\
& $R_{2}=0.45 \pm 0.15$ & $9.56_{-\infty}^{+0.31}$ \\
& $R_{3}=0.40 \pm 0.07$ & $9.56_{-0.41}^{+0.26}$ \\
\hline
\end{tabular}

${ }^{\mathrm{a}} R_{1}=f_{1400} / f_{1401}, R_{2}=f_{1400} / f_{1405}$, and $R_{3}=f_{1405} / f_{1401}$.

electron densities at $\log T=5.14$ of $\log n_{e}=9.65 \pm 0.20$ and $\log n_{e}=9.50 \pm 0.30$ for $\alpha$ Cen A and $\alpha$ Cen B, respectively, and pressures (in units of $\mathrm{cm}^{-3} \mathrm{~K}$ ) of $\log P_{e}=14.79$ \pm 0.20 and $\log P_{e}=14.64 \pm 0.30$.

For comparison, the atmospheric models of $\alpha$ Cen A and B created by Jordan et al. (1987) ffrom IUE and Einstein data have transition region pressures of $\log P_{e}=14.80$ and $\log P_{e}=15.28$, respectively. Their $\alpha$ Cen A value agrees very well with our results, but their $\alpha$ Cen B pressure is about a factor of 4 higher than what we derive. Based on observations of $\alpha$ Cen made by the Extreme Ultraviolet Explorer (EUVE), Mewe et al. (1995) used line ratios of $\mathrm{Fe} \mathrm{x}-\mathrm{Fe}$ xIv lines to derive a best estimate for the electron density at about $\log T=6.15$ of $\log n_{e} \approx 8.7 \pm 0.5$, which corresponds to a coronal pressure of $\log P_{e} \approx 14.85 \pm 0.50$. Since EUVE cannot resolve the two stars of the $\alpha$ Cen system, this pressure represents an average for the two stars. The inferred coronal pressure is consistent with the transition region pressures we obtained from the $\mathrm{O}$ IV] lines. In contrast, based on similar comparisons of pressures derived from $E U V E$ and O rv] data, Procyon and Capella appear to have coronal pressures that are significantly higher than their transition region pressures (Wood et al. 1996; Schmitt, Haisch, \& Drake 1994; Linsky et al. 1995; Dupree et al. 1993). This discrepancy may indicate that the line ratio diagnostics for the coronal plasma are unreliable, or it may indicate that on Procyon and Capella, the transition region line emission is to a large extent arising from regions physically unconnected with those responsible for the coronal $\mathrm{X}$-ray and EUV emission. Apparently, this does not have to be the case for $\alpha$ Cen A and B.

\section{THE C IV AND Si IV LINES OF 31 COMAE, $\beta$ CETI, $\beta$ DRACONIS, AND $\beta$ GEMINORUM}

\subsection{Data Analysis}

For 31 Com, $\beta$ Cet, $\beta$ Dra, and $\beta$ Gem, many spectral regions were observed with the GHRS, but in this paper we will confine our attention to the $\mathrm{C}$ IV and $\mathrm{Si}$ IV lines. We fitted these lines with Gaussians, as we did for the Si IV lines of $\alpha$ Cen A and B in $\S 3.1$. In addition to correcting for instrumental broadening, we also compensated for rotational broadening for $31 \mathrm{Com}$ and $\beta$ Dra, because both stars have $v \sin i$ values that are large compared with the spectral resolutions of the observations (see Tables 1 and 2). The parameters of the single-Gaussian fits are given in Table 6. The velocities in the table are in the stellar rest frames, based on the radial velocities listed in Table 1. For $\beta$ Gem, the single-Gaussian fits, illustrated in Figure 5, are acceptable within the $2 \sigma$ criterion. However, that is not the case for the single-Gaussian fits to the Si IV and C rv lines of the other three stars. Thus, the Si IV and C IV lines of 31 Com, $\beta$ Cet, and $\beta$ Dra were modeled with two Gaussians, as depicted in Figures 6,7, and 8. The parameters of the fits are given in Table 7 .

In order to reduce the number of free parameters, we fitted the two C IV lines simultaneously, which makes the parameters of the weaker line dependent on those of the stronger line. For $31 \mathrm{Com}$, this is particularly useful because the two $\mathrm{C}$ iv lines are partially blended. By fitting the lines simultaneously and assuming the two lines have essentially the same profiles, the uncontaminated red wing of the weaker line constrains the shape of the red wing of the stronger line, and the uncontaminated blue wing of the stronger line constrains the shape of the blue wing of the weaker line.

For $\beta$ Ceti, fitting the lines simultaneously is also very useful because the two $C$ iv lines are blended with at least

TABLE 6

Single-Gaussian Fit Parameters for 31 Com, $\beta$ Cet, $\beta$ Dra, and $\beta$ Gem

\begin{tabular}{|c|c|c|c|c|c|c|c|}
\hline Star & Ion & $\begin{array}{l}\lambda_{\text {rest }} \\
(\AA)\end{array}$ & $\begin{array}{l}\lambda_{\text {meas }} \\
(\AA)\end{array}$ & $\begin{array}{c}v^{\mathrm{a}} \\
\left(\mathrm{km} \mathrm{s}^{-1}\right)\end{array}$ & $\begin{array}{c}f \\
\left(10^{-13}\right)\end{array}$ & $\begin{array}{l}\text { FWHM } \\
\left(\mathrm{km} \mathrm{s}^{-1}\right)\end{array}$ & $\chi_{v}^{2}$ \\
\hline$\beta$ Cet . & Si rv & 1393.755 & 1393.874 & $13 \pm 1$ & $6.55 \pm 0.06$ & $103 \pm 1$ & 5.206 \\
\hline$\beta$ Cet ... & $\mathrm{Si} \mathrm{IV}$ & 1402.770 & 1402.880 & $10 \pm 1$ & $3.29 \pm 0.04$ & $100 \pm 1$ & 2.414 \\
\hline$\beta$ Cet $\ldots$. & $\mathrm{CIV}$ & 1548.202 & 1548.347 & $15 \pm 1$ & $5.50 \pm 0.09$ & $106 \pm 2$ & 1.892 \\
\hline$\beta$ Cet & C IV & 1550.774 & 1550.912 & $14 \pm 1$ & $2.82 \pm 0.07$ & $98 \pm 2$ & 1.416 \\
\hline 31 Com & Si rv & 1393.755 & 1393.779 & $6 \pm 1$ & $4.70 \pm 0.04$ & $196 \pm 2$ & 1.829 \\
\hline 31 Com. & Si rv & 1402.770 & 1402.764 & $0 \pm 1$ & $2.59 \pm 0.03$ & $191 \pm 3$ & 1.309 \\
\hline 31 Com .... & $\mathrm{C}_{\text {IV }}$ & 1548.202 & 1548.213 & $3 \pm 1$ & $12.5 \pm 0.1$ & $240 \pm 3$ & 1.800 \\
\hline $31 \mathrm{Com}$. & $\mathrm{C}$ IV & 1550.774 & 1550.815 & $9 \pm 2$ & $6.4 \pm 0.1$ & $207 \pm 4$ & 1.800 \\
\hline$\beta$ Dra $(1995)$ & Si rv & 1393.755 & 1393.779 & $25 \pm 1$ & $7.68 \pm 0.08$ & $158 \pm 2$ & 1.568 \\
\hline$\beta$ Dra (1995). & Si rv & 1402.770 & 1402.734 & $13 \pm 1$ & $4.51 \pm 0.07$ & $153 \pm 2$ & 1.204 \\
\hline$\beta$ Dra (1992) ...... & $\mathrm{C}_{\text {IV }}$ & 1548.202 & 1548.125 & $5 \pm 1$ & $16.4 \pm 0.2$ & $195 \pm 2$ & 1.898 \\
\hline$\beta$ Dra (1992). & C IV & 1550.774 & 1550.753 & $16 \pm 1$ & $9.0 \pm 0.1$ & $152 \pm 3$ & 1.898 \\
\hline$\beta$ Dra (1993). & $\mathrm{C}$ IV & 1548.202 & 1548.146 & $9 \pm 1$ & $16.7 \pm 0.2$ & $184 \pm 2$ & 1.355 \\
\hline$\beta$ Dra (1993). & $\mathrm{C}$ rV & 1550.774 & 1550.775 & $20 \pm 1$ & $8.8 \pm 0.1$ & $141 \pm 3$ & 1.355 \\
\hline$\beta$ Dra $(1995) \ldots . .$. & $\mathrm{C} \mathrm{IV}$ & 1548.202 & 1548.187 & $17 \pm 1$ & $13.4 \pm 0.2$ & $183 \pm 2$ & 1.521 \\
\hline$\beta$ Dra $(1995) \ldots \ldots$ & $\mathrm{CIV}$ & 1550.774 & 1550.788 & $23 \pm 1$ & $7.8 \pm 0.1$ & $148 \pm 3$ & 1.521 \\
\hline 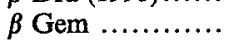 & Si rv & 1393.755 & 1393.816 & $10 \pm 1$ & $1.44 \pm 0.04$ & $82 \pm 2$ & 1.250 \\
\hline$\beta \mathrm{Gem}$ & $\mathrm{Si} \mathbf{I V}$ & 1402.770 & 1402.841 & $12 \pm 1$ & $0.81 \pm 0.03$ & $80 \pm 3$ & 1.054 \\
\hline$\beta \mathrm{Gem} . .$. & $\mathrm{C}$ IV & 1548.202 & 1548.300 & $16 \pm 1$ & $1.63 \pm 0.07$ & $90 \pm 3$ & 1.133 \\
\hline$\beta$ Gem ..... & $\mathrm{C}$ IV & 1550.774 & 1550.859 & $13 \pm 1$ & $0.82 \pm 0.03$ & $74 \pm 3$ & 1.219 \\
\hline
\end{tabular}

a The line velocity relative to the star. 


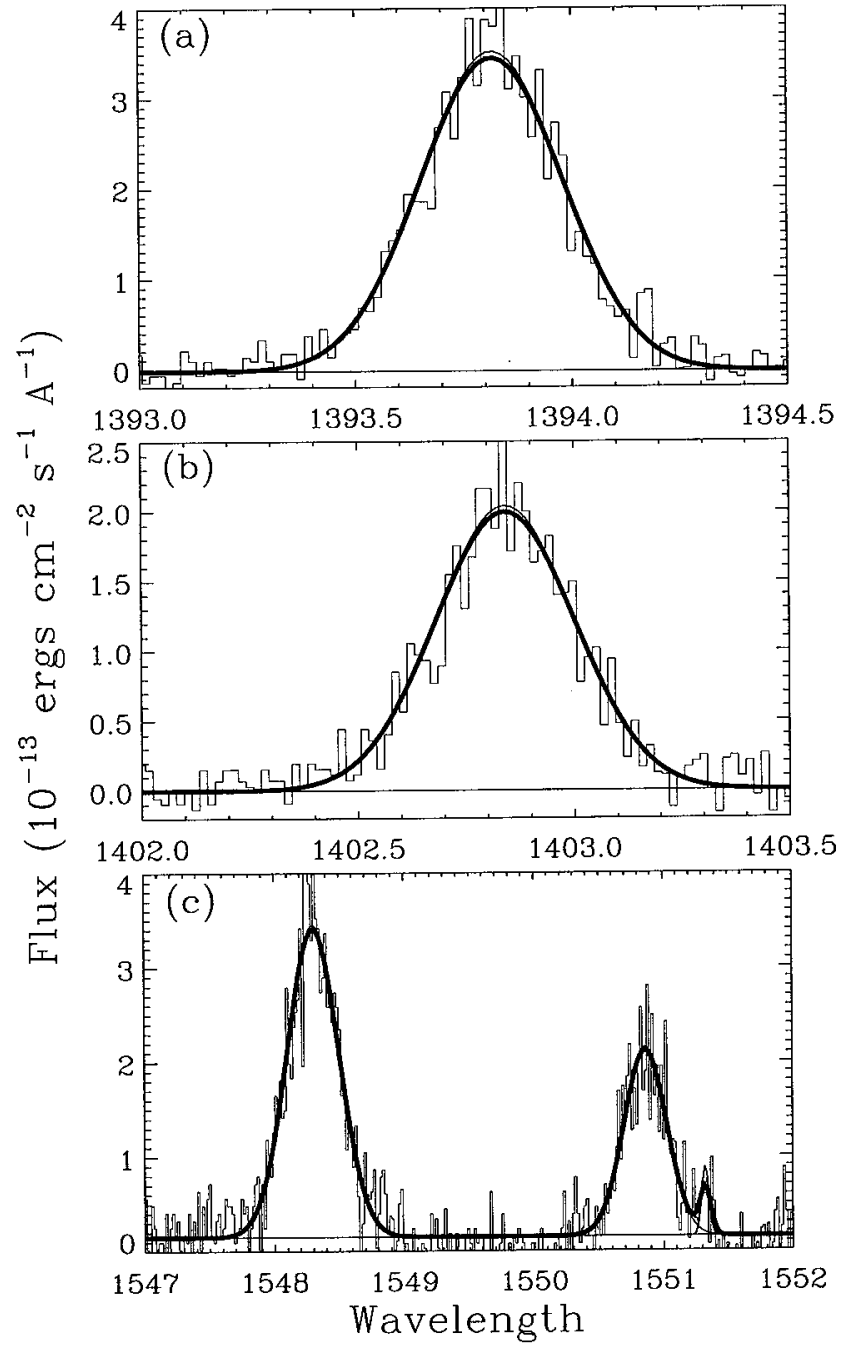

FIG. 5.-Single-Gaussian fits to the Si Iv and C rv lines of $\beta \mathrm{Gem}$. The parameters of these fits are given in Table 6. The Gaussian fit is shown before (narrow solid line) and after (thick solid line) convolution with the instrumental profile. The emission lines shown are $(a)$ the Si rv $\lambda 1394$ line, (b) the Si IV $\lambda 1403$ line, and (c) the C IV $\lambda \lambda 1548,1551$ lines. A Si I line in the red wing of the C IV $\lambda 1551$ line is also fitted with a Gaussian.

three Si I emission features, which are fitted simultaneously with the $\mathrm{C}$ IV lines (see Fig. $6 c$ ). The Si I features, which were identified using the solar spectrum (Sandlin et al. 1986), obscure the far blue wing of the stronger line and the red wing of the weaker line. Fitting the $\mathrm{C}$ Iv lines simultaneously and assuming the two lines have essentially the same profile produces a more accurate separation of the $\mathrm{C}$ IV lines from the $\mathrm{Si}$ I lines. (Note that the $\mathrm{Si}$ I line at $1551.3 \AA$ is also seen and corrected for in the $\beta$ Gem spectrum shown in Fig. $5 c$.)

When the C Iv lines of HR 1099 and the Si Iv lines of $\alpha$ Cen B were fitted simultaneously by Wood et al.(1996) and in $\S 3.1$, respectively, the number of free parameters was reduced by forcing the widths and velocities of the narrow and broad components to be the same for both lines and by forcing the $\lambda 1548 / \lambda 1551$ and $\lambda 1394 / \lambda 1403$ flux ratios to be the optically thin value of 2 . In other words, the two line profiles were assumed to be identical with the stronger line having exactly twice the flux of the weaker line. Unfortunately, we cannot assume this for the $\mathrm{C}$ IV lines of $31 \mathrm{Com}$ and $\beta$ Dra because the single-Gaussian fit parameters

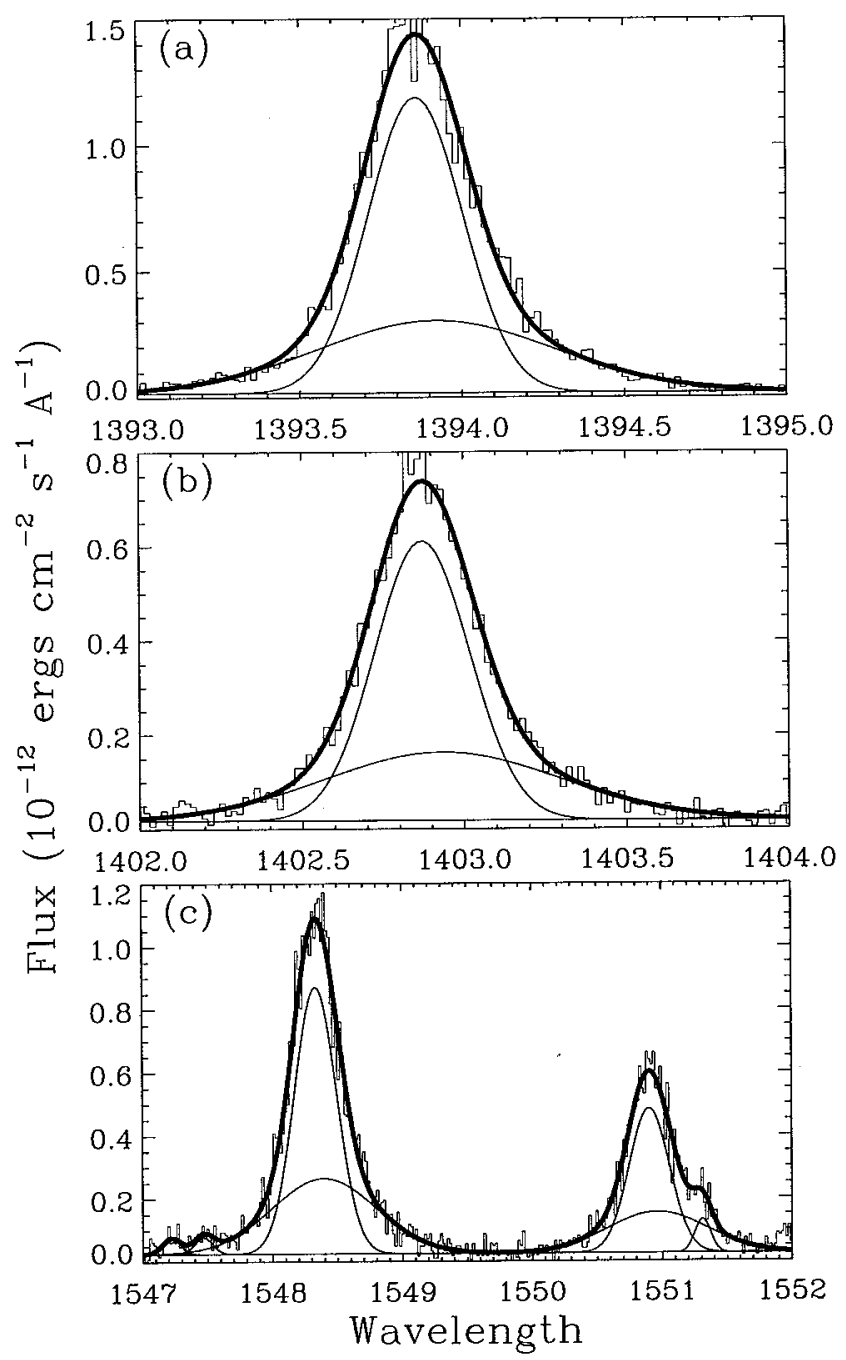

Fig. 6.-Two-Gaussian fits to the Si Iv and $\mathrm{C}$ rv lines of $\beta$ Cet. The parameters of these fits are given in Table 7. For each fit, the Gaussian components are plotted as thin solid lines, and the convolution of the sum of the components with the instrumental profile is shown as a thick solid line. The emission lines shown are (a) the Si IV $\lambda 1394$ line, $(b)$ the Si IV $\lambda 1403$ line, and $(c)$ the $C$ IV $\lambda \lambda 1548,1551$ lines. Two Si I features in the blue wing of the $C_{\text {IV }} \lambda 1548$ line and an additional $\mathrm{Si}$ I line in the red wing of $\mathrm{C}$ IV $\lambda 1551$ are also fit with Gaussians.

clearly demonstrate that the line profiles are not identical. The stronger $C$ iv line is broader and has a smaller redshift than the weaker line, and for $\beta$ Dra the $\lambda 1548 / \lambda 1551$ flux ratio is significantly less than 2 . Linsky et al. (1995) found that the C Iv lines of Capella have similar properties. The only way we can explain these properties of the $\mathrm{C}$ IV lines is to appeal to opacity effects and/or unidentified line blends. The implications of this conclusion will be discussed further in $\$ \$ 4.2$ and 5 .

Thus, for the two-Gaussian fits to the $\mathrm{C}$ IV lines of 31 Com and $\beta$ Dra, we allowed the velocities of the $\lambda 1551$ narrow and broad components to be different from the velocities of the $\lambda 1548$ narrow and broad components, but we constrained the fit by forcing the velocity difference between the two broad components to be the same as the velocity difference between the narrow components. Similarly, we allowed the widths of the $\lambda 1551$ narrow and broad components to be different from the widths of the $\lambda 1548$ narrow and broad components, but we forced the 

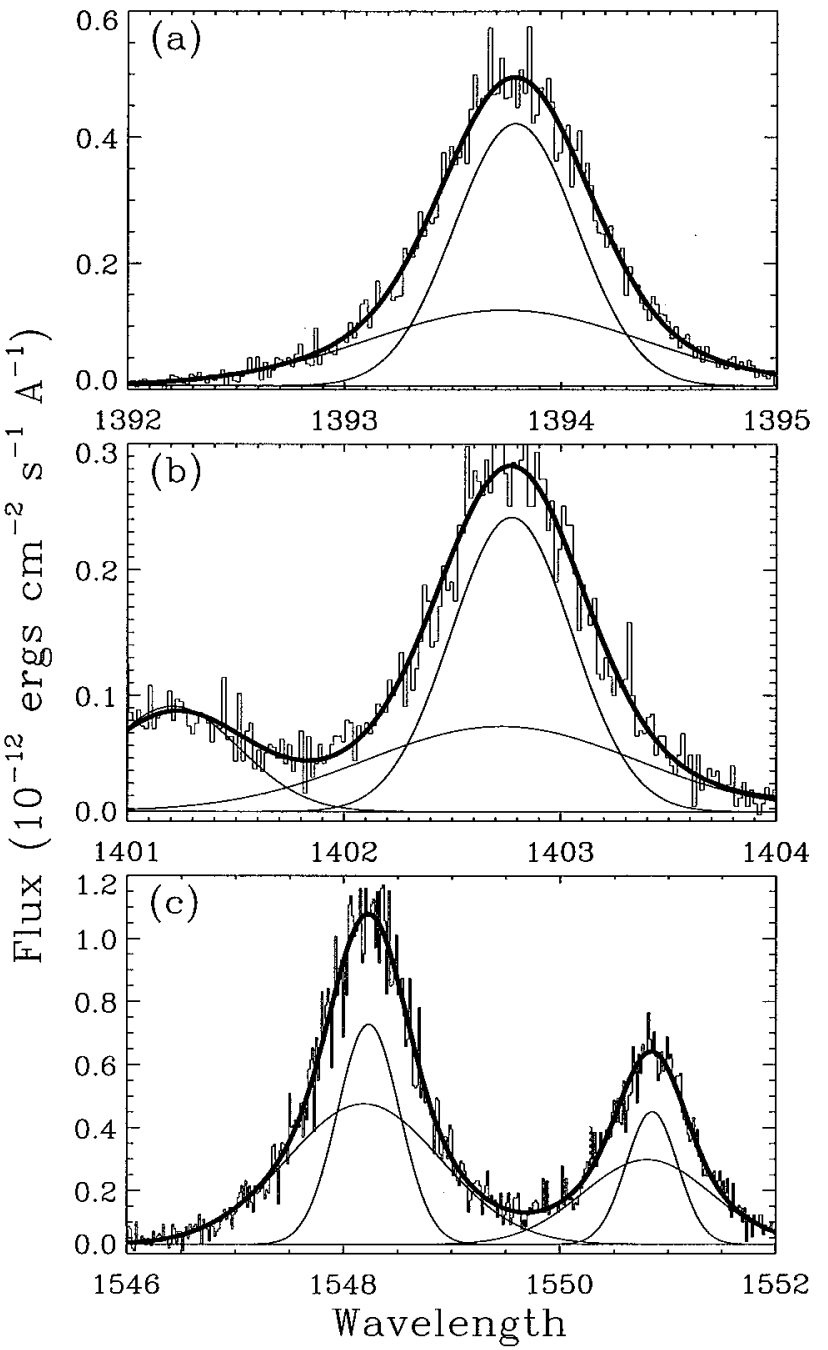

Fig. 7.-Two-Gaussian fits to the Si Iv and $\mathrm{C}$ iv lines of $31 \mathrm{Com}$. The parameters of these fits are given in Table 7. For each fit, the Gaussian components are plotted as thin solid lines, and the convolution of the sum of the components with the instrumental and rotational broadening profiles is shown as a thick solid line. The emission lines shown are (a) the $\mathrm{Si} \mathrm{rv}$ $\lambda 1394$ line, $(b)$ the Si IV $\lambda 1403$ line, and $(c)$ the $C$ IV $\lambda \lambda 1548,1551$ lines. The O IV] $\lambda 1401$ line is visible in the blue wing of the Si IV $\lambda 1403$ line, and it has also been fitted with a Gaussian.

FWHM $_{\lambda 1551} / \mathrm{FWHM}_{\lambda 1548}$ ratio to be the same for both the narrow and broad components. Also, we required the $\lambda 1548 / \lambda 1551$ flux ratio to be the same for both the broad and narrow components, although we allowed this ratio to be different from 2. Although there is no physical basis for these assumptions, they allowed us to lower the number of free parameters from 12 to 9 , and the resulting fits are still excellent.

Partially because of the $\mathrm{Si}$ I blends, it was not immediately apparent whether the $\mathrm{C}$ IV lines of $\beta$ Cet have the same profile or if the two lines are significantly different, like the $\mathrm{C}$ IV lines of Capella, $31 \mathrm{Com}$, and $\beta$ Dra. Thus, we fitted the $C_{\text {IV }}$ lines of $\beta$ Cet in the same manner as the C IV lines of $31 \mathrm{Com}$ and $\beta$ Dra. The resulting parameters in Table 7 demonstrate that for $\beta$ Cet, the two C IV lines are essentially identical and have a flux ratio very close to 2 .

The two Si Iv lines of $\beta$ Cet and $\beta$ Dra were fitted simultaneously in the same manner as the two $C$ IV lines. For 31 Com, however, the $\lambda 1403$ line is blended with the $\mathrm{O}$ rv] lines

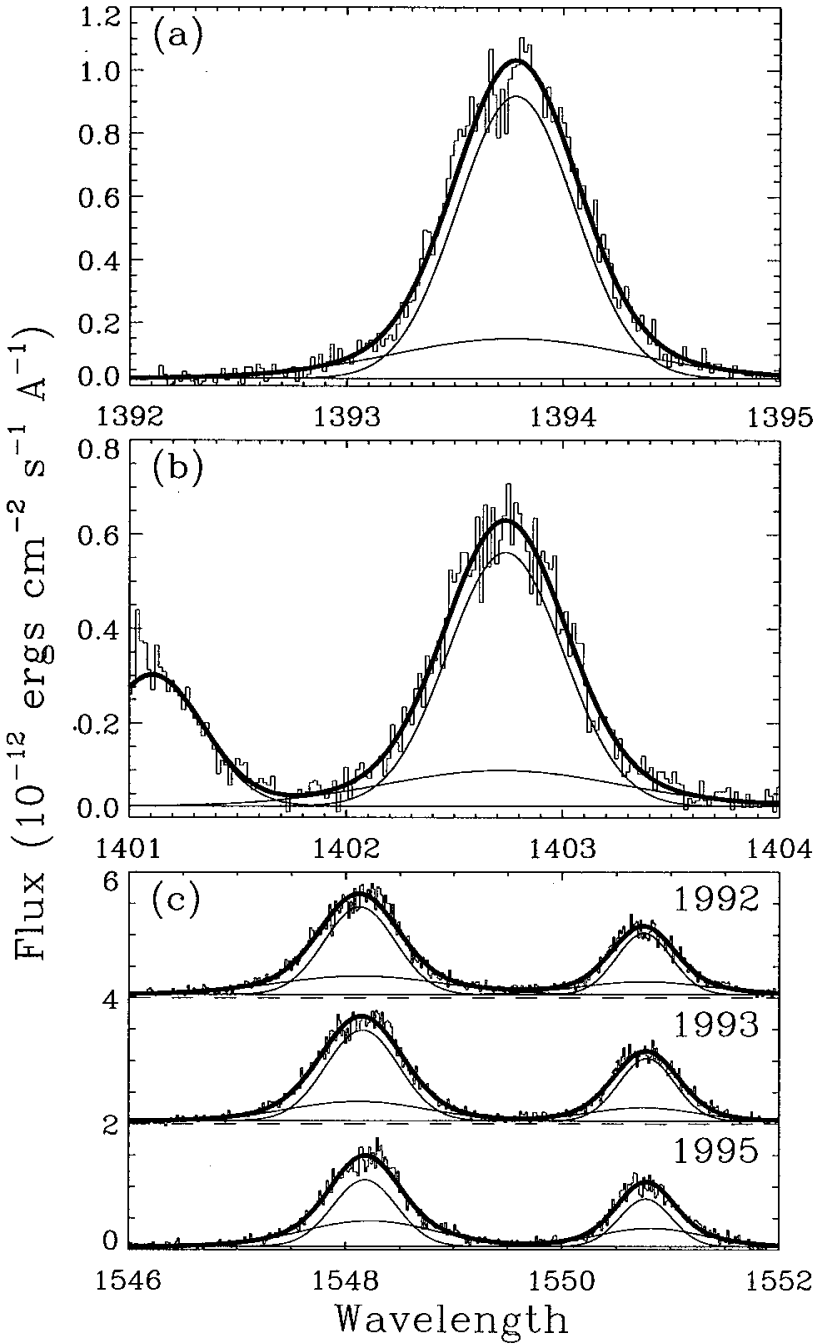

Fig. 8.-Two-Gaussian fits to the Si IV and $\mathrm{C}$ iv lines of $\beta$ Dra. The parameters of these fits are given in Table 7. For each fit, the Gaussian components are plotted as thin solid lines, and the convolution of the sum of the components with the instrumental and rotational broadening profiles is shown as a thick solid line. The emission lines shown are (a) the Si rv $\lambda 1394$ line, $(b)$ the Si rv $\lambda 1403$ line, and (c) the C rv $\lambda \lambda$ 1548, 1551 lines observed in three different years, where the dashed lines indicate the zero flux levels for each spectrum. The $\mathrm{O}$ Iv] $\lambda 1401$ line is visible in the blue wing of the Si Iv $\lambda 1403$ line, and it has also been fitted with a Gaussian.

(especially the $\lambda 1401$ line; see Fig. $7 b$ ) to the extent that we decided to fit the uncontaminated $\lambda 1394$ line independently of the $\lambda 1403$ line. The $\lambda 1403$ line was then fitted simultaneously with the blended O IV] lines, and the narrow and broad component parameters of the $\lambda 1403$ line were constrained by the previously measured $\lambda 1394$ parameters in a manner analogous to that described above for the $\mathrm{C} \mathrm{IV}$ lines.

\subsection{The Variable $C_{\text {IV }}$ Lines of $\beta$ Draconis}

$H S T$ first observed $\beta$ Dra on 1992 April 23. Two of the planned GHRS observations were G160M exposures of the 1383-1419 $\AA$ and $1532-1568 \AA$ spectral intervals, the former containing the $\mathrm{Si}$ Iv lines and the latter containing the $\mathrm{C}$ IV lines. However, the Si IV observation failed owing to a malfunction of the GHRS grating carrousel, although the other observations were completed successfully. In order to complete the data set, $\beta$ Dra was observed again about a year later on 1993 February 20. In addition to the missing Si IV 
TABLE 7

Two-Gaussian Fit Parameters for 31 Com, $\beta$ Cet, and $\beta$ Dra

\begin{tabular}{|c|c|c|c|c|c|c|c|}
\hline Star & Ion & $\begin{array}{l}\lambda_{\text {rest }} \\
(\AA)\end{array}$ & $\begin{array}{c}\lambda_{\text {meas }} \\
(\AA)\end{array}$ & $\begin{array}{c}v^{\mathrm{a}} \\
\left(\mathrm{km} \mathrm{s}^{-1}\right)\end{array}$ & $\begin{array}{c}f \\
\left(10^{-13}\right)\end{array}$ & $\begin{array}{l}\text { FWHM } \\
\left(\mathrm{km} \mathrm{s}^{-1}\right)\end{array}$ & $\chi_{v}^{2}$ \\
\hline \multirow[t]{2}{*}{$\beta$ Cet $\ldots \ldots \ldots \ldots \ldots$} & Si IV & 1393.755 & 1393.859 & $9 \pm 1$ & $4.3 \pm 0.2$ & $74 \pm 2$ & 1.271 \\
\hline & & 1393.755 & 1393.920 & $22 \pm 2$ & $2.6 \pm 0.2$ & $185 \pm 6$ & 1.271 \\
\hline \multirow[t]{2}{*}{$\beta$ Cet $\ldots \ldots \ldots \ldots \ldots$} & Si IV & 1402.770 & 1402.871 & $9 \pm 1$ & $2.18 \pm 0.08$ & $73 \pm 2$ & 1.271 \\
\hline & & 1402.770 & 1402.932 & $22 \pm 2$ & $1.32 \pm 0.08$ & $181 \pm 6$ & 1.271 \\
\hline \multirow[t]{2}{*}{$\beta$ Cet $\ldots \ldots \ldots \ldots \ldots$} & C IV & 1548.202 & 1548.332 & $12 \pm 1$ & $3.4 \pm 0.2$ & $74 \pm 2$ & 1.047 \\
\hline & & 1548.202 & 1548.395 & $24 \pm 3$ & $2.4 \pm 0.2$ & $183 \pm 9$ & 1.047 \\
\hline \multirow{2}{*}{$\beta$ Cet $\ldots \ldots \ldots \ldots \ldots$} & $\mathrm{Crv}$ & 1550.774 & 1550.901 & $11 \pm 1$ & $1.8 \pm 0.1$ & $70 \pm 2$ & 1.047 \\
\hline & & 1550.774 & 1550.963 & $23 \pm 3$ & $1.2 \pm 0.1$ & $175 \pm 11$ & 1.047 \\
\hline \multirow{2}{*}{$31 \mathrm{Com} . . . \ldots \ldots \ldots$} & Si IV & 1393.755 & 1393.789 & $8 \pm 2$ & $3.0 \pm 0.3$ & $144 \pm 7$ & 1.040 \\
\hline & & 1393.755 & 1393.742 & $-2 \pm 5$ & $1.9 \pm 0.3$ & $321 \pm 21$ & 1.040 \\
\hline \multirow[t]{2}{*}{$31 \mathrm{Com} . . . \ldots \ldots$} & Si rv & 1402.770 & 1402.773 & $2 \pm 2$ & $1.6 \pm 0.1$ & $141 \pm 8$ & 1.006 \\
\hline & & 1402.770 & 1402.726 & $-8 \pm 5$ & $1.1 \pm 0.1$ & $314 \pm 22$ & 1.006 \\
\hline \multirow{2}{*}{31 Com ............ } & $\mathrm{CIV}$ & 1548.202 & 1548.232 & $7 \pm 2$ & $5.0 \pm 0.6$ & $131 \pm 9$ & 1.153 \\
\hline & & 1548.202 & 1548.188 & $-2 \pm 3$ & $8.0 \pm 0.5$ & $325 \pm 13$ & 1.153 \\
\hline \multirow{2}{*}{$31 \mathrm{Com} . . . \ldots \ldots$} & $\mathrm{C}$ IV & 1550.774 & 1550.849 & $16 \pm 2$ & $2.5 \pm 0.3$ & $109 \pm 8$ & 1.153 \\
\hline & & 1550.774 & 1550.805 & $7 \pm 4$ & $4.0 \pm 0.3$ & $270 \pm 11$ & 1.153 \\
\hline \multirow{2}{*}{$\beta$ Dra $(1995) \ldots \ldots$} & Si IV & 1393.755 & 1393.782 & $26 \pm 1$ & $6.1 \pm 0.4$ & $137 \pm 4$ & 1.045 \\
\hline & & 1393.755 & 1393.765 & $22 \pm 6$ & $1.8 \pm 0.4$ & $282 \pm 27$ & 1.045 \\
\hline \multirow[t]{2}{*}{$\beta$ Dra $(1995) \ldots \ldots$} & Si IV & 1402.770 & 1402.736 & $13 \pm 1$ & $3.6 \pm 0.2$ & $134 \pm 4$ & 1.045 \\
\hline & & 1402.770 & 1402.719 & $9 \pm 6$ & $1.0 \pm 0.2$ & $277 \pm 26$ & 1.045 \\
\hline \multirow[t]{2}{*}{$\beta$ Dra (1992)...... } & $\mathrm{C}_{\text {IV }}$ & 1548.202 & 1548.128 & $6 \pm 1$ & $11.4 \pm 0.8$ & $150 \pm 6$ & 1.240 \\
\hline & & 1548.202 & 1548.132 & $7 \pm 6$ & $5.7 \pm 0.8$ & $353 \pm 30$ & 1.240 \\
\hline \multirow[t]{2}{*}{$\beta$ Dra $(1992) \ldots \ldots$} & $\mathrm{C}$ rv & 1550.774 & 1550.752 & $16 \pm 2$ & $6.2 \pm 0.5$ & $115 \pm 5$ & 1.240 \\
\hline & & 1550.774 & 1550.756 & $17 \pm 6$ & $3.1 \pm 0.4$ & $272 \pm 23$ & 1.240 \\
\hline \multirow[t]{2}{*}{$\beta$ Dra (1993)...... } & $\mathrm{C} \mathbf{I V}$ & 1548.202 & 1548.154 & $11 \pm 1$ & $12.1 \pm 1.3$ & $153 \pm 7$ & 1.149 \\
\hline & & 1548.202 & 1548.113 & $3 \pm 6$ & $4.9 \pm 1.2$ & $291 \pm 27$ & 1.149 \\
\hline \multirow[t]{2}{*}{$\beta$ Dra (1993)...... } & $\mathrm{C}$ IV & 1550.774 & 1550.780 & $21 \pm 2$ & $6.4 \pm 0.7$ & $117 \pm 5$ & 1.149 \\
\hline & & 1550.774 & 1550.738 & $13 \pm 6$ & $2.6 \pm 0.6$ & $223 \pm 20$ & 1.149 \\
\hline \multirow[t]{2}{*}{$\beta$ Dra $(1995) \ldots \ldots$} & C IV & 1548.202 & 1548.178 & $16 \pm 2$ & $7.8 \pm 0.8$ & $134 \pm 7$ & 1.136 \\
\hline & & 1548.202 & 1548.221 & $24 \pm 4$ & $6.2 \pm 0.8$ & $277 \pm 18$ & 1.136 \\
\hline \multirow{2}{*}{$\beta$ Dra $(1995) \ldots \ldots$} & C IV & 1550.774 & 1550.770 & $20 \pm 2$ & $4.5 \pm 0.5$ & $109 \pm 5$ & 1.136 \\
\hline & & 1550.774 & 1550.814 & $28 \pm 4$ & $3.6 \pm 0.5$ & $224 \pm 14$ & 1.136 \\
\hline
\end{tabular}

a The line velocity relative to the star.

spectrum, another C IV spectrum also was planned. Although the C Iv spectrum was obtained successfully, the $\mathrm{Si}$ IV observation failed again due to yet another carrousel malfunction. On 1995 April 29, $\beta$ Dra was acquired for a third time by HST for observations of Si IV and C IV, and finally both spectra were obtained without incident. One positive result of the repeated failure of the $\mathrm{Si}$ IV observation is that GHRS spectra of the $\mathrm{C}$ IV lines were acquired in three different years, which allowed us to check for line profile variability.

Figure 9 illustrates the smoothed C IV profiles for all three years, and Figure $8 c$ shows the two-Gaussian fits to

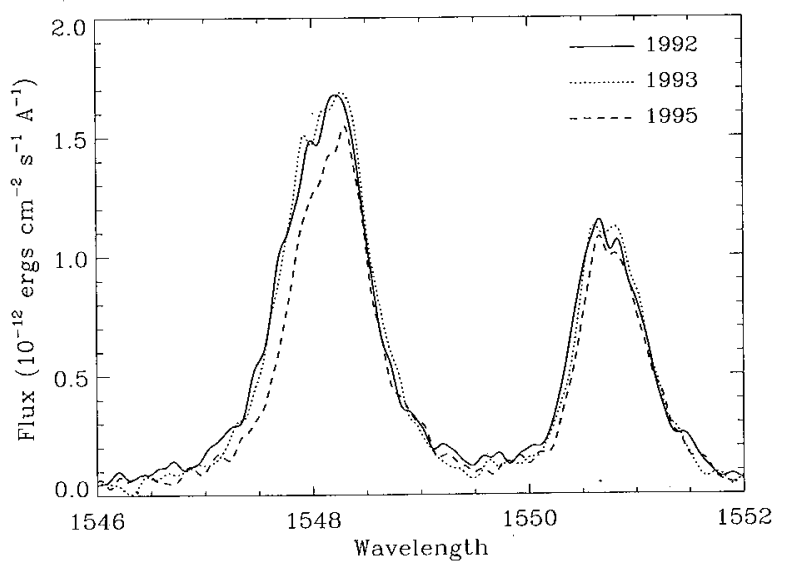

FIG. 9.-Fourier-smoothed spectra of the C Iv lines of $\beta$ Dra observed in three different years. Line profile variability is apparent. these lines. Line profile variability is evident in both Figure 9 and in the C IV parameters listed in Tables 6 and 7. The C Iv fluxes appear to be constant between 1992 and 1993, but the $\lambda 1548$ and $\lambda 1551$ fluxes dropped by $18 \%$ and $10 \%$, respectively, between 1993 and 1995. The effects of inaccuracies in the GHRS flux calibration must be considered here, because the 1992 and 1993 data are pre-COSTAR observations, while the 1995 spectrum was taken after the installation of COSTAR, which affected the instrumental sensitivities. However, flux calibration inaccuracies cannot explain why the flux decrease is different for the two C IV lines, so we believe the flux decrease is due to true stellar variability. Since the variability is more pronounced in the more optically thick C rv $\lambda 1548$ line, opacity effects probably play a significant role in altering the $\mathrm{C}$ IV profiles.

In addition to the flux change, the $1995 \mathrm{C}$ rv lines also are more redshifted than they were in 1992 and 1993, and once again the change is larger for the 21548 line. An increase in line redshift also is evident between 1992 and 1993. Quantitatively, the measured $\lambda 1548(\lambda 1551)$ line velocities based on the single-Gaussian fits are $5 \pm 1,9 \pm 1$, and $17 \pm 1$ $(16 \pm 1,20 \pm 1$, and $23 \pm 1)$ for the 1992, 1993, and 1995 data, respectively. The velocity variability is too large to be due to inaccuracies in the wavelength calibration.

The obvious velocity and flux variability, combined with more subtle line profile changes, naturally have affected the parameters of the two-Gaussian fits. For example, the wings of the 1992 C IV profiles appear to be slightly more extended than is the case for the other two years, resulting in wider broad components. Also, the upper halves of the $1995 \mathrm{C} \mathrm{IV}$ 
profiles are narrower than is the case for the other two years, whch results in thinner narrow components. The broad component contribution to the $\mathrm{C}$ IV lines also is significantly larger for the 1995 profiles than it is for the 1992 and 1993 profiles.

As discussed in $\S 1$, we currently are working under the hypothesis that the broad components are associated with microflaring. Thus, the larger broad component contribution to the $1995 \mathrm{C}$ IV profiles appears to suggest a greater prevalence of microflaring in 1995 than in 1992 and 1993. However, we mentioned above that opacity effects probably play some role in the line variability. Opacity effects and/or unidentified line blends also appear to be necessary in order to explain the general velocity, width, and flux discrepancies between the two $\mathrm{C}$ IV lines of $\beta$ Dra, Capella, and $31 \mathrm{Com}$ (see above). To some extent, these effects compromise the ability of the two-Gaussian fits to accurately quantify the narrow and broad component contributions to the line emission. We will return to this issue in $\S 5$.

With only three $\beta$ Dra C IV observations spaced so far apart in time, we cannot determine whether the detected variability is associated with different regions of the star rotating into and out of view, or whether the variability is more global, perhaps like that associated with the $11 \mathrm{yr}$ activity cycle of the Sun. Furthermore, because the profile changes primarily are on the blue sides of the C IV profiles, we cannot rule out a warm stellar wind as the source of the variability. The radius and $v \sin i$ values in Table 1 suggest that the rotational period of $\beta$ Dra is roughly 6 months, which is less than the time between the observations. Thus, rotational modulation is a plausible explanation.

\section{COMPARING STELLAR TRANSITION REGION LINE PROFILES}

\subsection{Broad Components and Microflaring}

The primary goal of this paper is to explore the variations of stellar transition region line profiles with activity and other stellar properties such as surface gravity. The transition region lines for ten of the stars in our sample are displayed in Figure 10 along with the Gaussian fits to the profiles. For each star, the line displayed is either Si IV $\lambda 1394$ or C IV $\lambda 1548$, depending on which is the highest quality transition region line profile available. For most of the stars, two Gaussians were required to fit the line profiles. For Capella, an additional third Gaussian was added to represent the emission from the G8 star of the binary system. As discussed in $\S 4.1$, a second Gaussian was not required to model the Si IV line of $\beta$ Gem accurately, so a single-Gaussian fit is displayed.

For Procyon, a simple single-Gaussian fit is used despite the obvious presence of a blue wing excess. The blue wing excess is seen for all of Procyon's transition region lines (Wood et al. 1996). However, except for Procyon's N v $\lambda 1239$ line, there is no corresponding red wing excess that would indicate the presence of a broad component like that seen for most of the stellar Si IV and C Iv lines in Figure 10. Attempts to fit two Gaussians to several of Procyon's transition region lines resulted in a reproduction of the singleGaussian fit plus a very small Gaussian in the blue wing of the line, rather than a narrow component plus a broad component. Wood et al. (1996) therefore concluded that Procyon's blue wing excesses have a different interpretation than the broad components observed in the transition region lines of the other stars. Since the single-Gaussian fits to Procyon's transition region lines are acceptable $2 \sigma$ fits when the blue wings are ignored, no two-Gaussian fits were presented, with the notable exception of $\mathrm{N} \mathrm{v} \lambda 1239$.

We converted previously published X-ray fluxes for our sample stars to surface fluxes using the stellar radii and distances listed in Table 1 . These X-ray surface fluxes are given in Table 8, together with the C IV surface fluxes (both lines combined). Both quantities will be considered to be measures of the stellar activity level in this paper. The stars in Table 8 are listed in order of decreasing X-ray surface flux, and the same order is used in Figure 10. Columns (4)-(9) of Table 8 list properties of the fits to the Si IV and $\mathrm{C}$ IV lines, and the last column indicates references for the quantities listed in the table (in addition to this paper). The ratio of the broad component flux to the total line flux is given in columns (4) and (5). The difference between the velocities of the broad and narrow components is given in columns (6) and (7). We converted the widths of the broad and narrow components to nonthermal velocities using the relation (in cgs units)

$$
\left(\frac{\Delta \lambda}{\lambda}\right)^{2}=3.08 \times 10^{-21}\left(\frac{2 k T}{m_{i}}+\xi^{2}\right),
$$

where $\Delta \lambda$ is the measured FWHM, $T$ is the line formation temperature, $m_{i}$ is the ion mass, and $\xi$ is the most probable nonthermal speed. The narrow and broad component nonthermal velocities listed in columns (8) and (9) of Table 8 represent weighted averages of all four Si IV and C IV lines.

Based on Figure 10, there appears to be a tendency for the transition region lines of active stars to have more nonGaussian profiles with broader wings than the less active stars. A particularly useful exercise is to compare the Si IV profiles of $\beta$ Cet and $\beta$ Gem (see also Figs. $5 a$ and $6 a$ ), because these two stars are very similar in all respects except activity level. The wings of the $\beta$ Cet Si Iv line clearly are more prominent than those of $\beta \mathrm{Gem}$.

The profiles in Figure 10 are compared more directly in Figure 11, in which the C IV profile of AB Dor from Vilhu et al. (1996) is also included. The profiles shown in Figure 11 are based on the two-Gaussian fits (except for $\beta$ Gem). The Procyon profile shown in Figure 11 is also based on a twoGaussian fit, because for the purposes of this figure we wanted the aforementioned blue-wing excess to be evident. The stellar line profiles in Figure 11 have been normalized to have the same amplitude and FWHM $(1.0$ and $100 \mathrm{~km}$ $\mathrm{s}^{-1}$, respectively), and at the half-maximum location we force the lines to be centered on $0 \mathrm{~km} \mathrm{~s}^{-1}$. A logarithmic scale for the flux is used to emphasize the line wings. The three stars with the largest X-ray surface flux (AU Mic, HR 1099 , and AB Dor) clearly have the broadest line wings, once again suggesting a correlation between activity and extended wing emission.

Based on Figures 10 and 11, we have argued that a correlation exists between stellar activity and broad transition region line wings. The question we now address is, what is the best way to quantify the prominence of the line wings? We propose two methods. The first is to measure the width of a profile at $5 \%$ of the maximum amplitude and then to divide this width by the FWHM. In Figures $12 a-12 c$, we plot this ratio against three traditional activity diagnostics for the chromosphere, transition region, and corona: the $\mathrm{Mg}$ II, C IV, and X-ray surface fluxes, respectively. The $\mathrm{Mg}$ II 


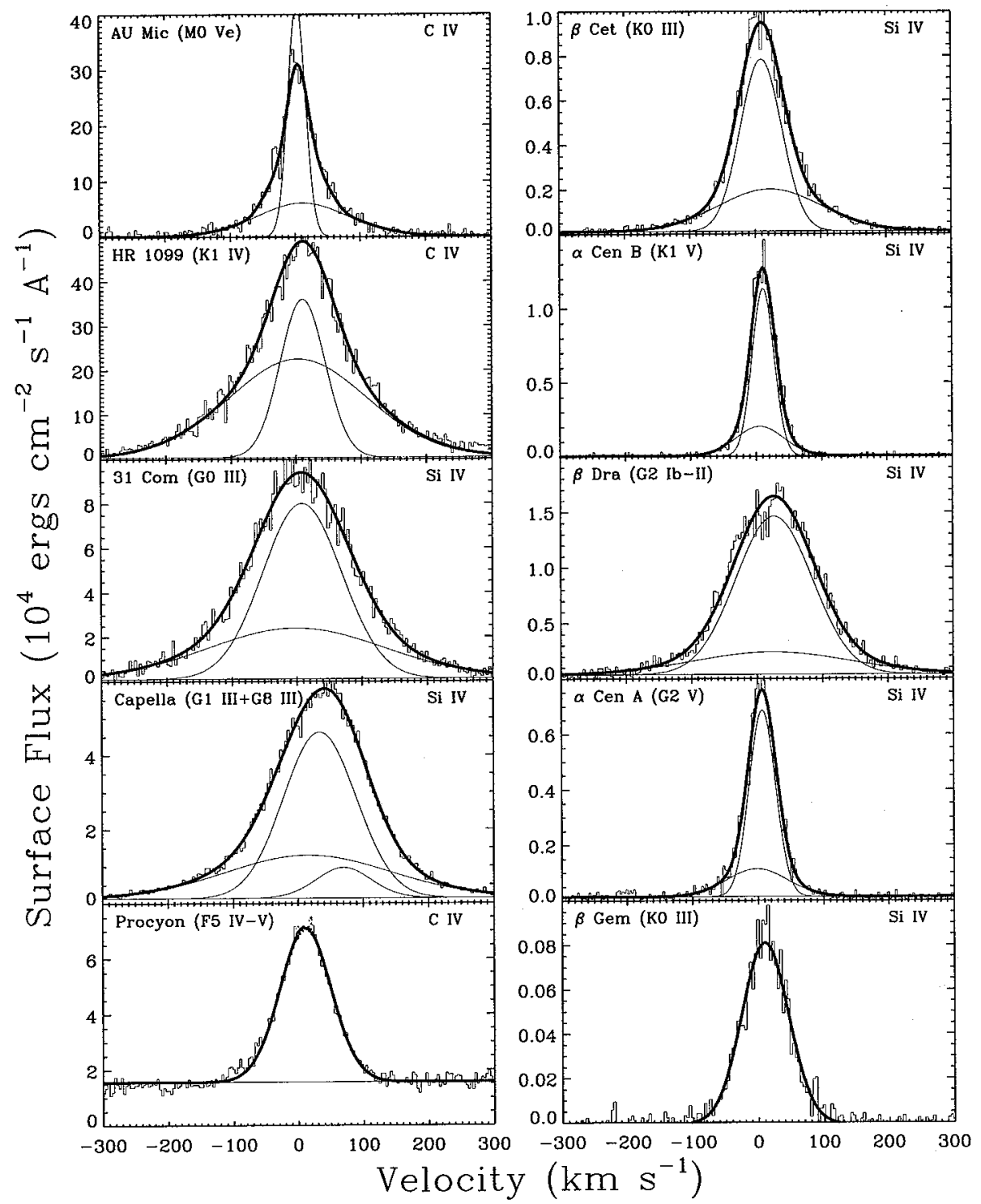

Fig. 10.-Our best Gaussian fits to the Si $\mathrm{Nv}$ or $\mathrm{Crv}$ lines of nearly all the stars in our sample. The emission line shown is either the Si rv $\lambda 1394$ line or the Crv $\lambda 1548$ line, depending on which is the most free of blends and has the highest $\mathbf{S} / \mathbf{N}$. All of these fits have already been displayed before, either earlier in this paper or in previous papers (Linsky \& Wood 1994; Linsky et al. 1995; Wood et al. 1996; Wood 1996), but here we show them all in one place to allow for easier comparison. With the exception of Procyon and $\beta$ Gem, two Gaussians are required to fit the data adequately. For Capella, a third Gaussian representing emission from the G8 III star is also included. The stars are placed in order of decreasing X-ray surface flux, reading from AU Mic to Procyon and then down from $\beta$ Cet to $\beta$ Gem.

fluxes were measured from GHRS data, except for the AB Dor value which is from IUE data (Rucinski 1985). Basal fluxes from Rutten et al. (1991) have been subtracted from the $\mathrm{Mg}$ II fluxes. The second method of quantifying the prominence of the line wings is to divide the broad component flux by the total line flux. These values, which are listed in columns (4) and (5) of Table 8 for both Si IV and $\mathrm{C}$ IV, are plotted versus the $\mathrm{Mg}$ II, $\mathrm{C}$ IV, and X-ray surface fluxes in Figures $12 d-12 f$.

Figures $12 c$ and $12 d$ clearly indicate a correlation between broad line wings and stellar activity. This correlation is less obvious in Figures $12 b$ and $12 e$ and is not very apparent at all in Figures $12 a$ and $12 d$. The reason that the correlation is most apparent when $F_{\mathrm{X}}$ is used as the activity diagnostic is probably that the $F_{\mathrm{X}}$ values vary by about 5 orders of magnitude, while the $F_{\mathrm{Mg} \text { II }}$ and $F_{\mathrm{CIV}}$ values vary by only about 2 and 3 orders of magnitude, respectively.
Thus, scatter in the data points produced by systematic errors can hide the correlation in the $\mathrm{Mg}$ II and $\mathrm{C}$ IV plots more easily than in the X-ray plots. Variations in line profile behavior with spectral type are probably the largest source of scatter in Figure 12. In determining how line profiles vary with activity, ideally one should observe stars with the same spectral type but different activity levels. Unfortunately, such a data set is not available at this time, so our sample necessarily contains cool stars with a large range of spectral types. Other causes of the scatter in Figure 12 include uncertainties in $F_{\mathrm{Mg} \mathrm{II}}, F_{\mathrm{CIV}}$, and $F_{\mathrm{X}}$ due to both stellar variability and uncertainties in stellar radii and distances, line profile variability such as that seen for $\beta$ Dra, and uncertainties introduced into our analysis by opacity effects and line blending, as discussed in $\S 4.2$.

The upper limit representing Procyon in Figures $12 d-12 f$ appears to be inconsistent with the general trend implied by 
TABLE 8

Transition Region Line Profile Properties

\begin{tabular}{|c|c|c|c|c|c|c|c|c|c|}
\hline \multirow[b]{2}{*}{$\begin{array}{c}\text { StaR } \\
\text { (1) }\end{array}$} & \multirow[b]{2}{*}{$\underset{\text { (2) }}{\log F_{X}}$} & \multirow[b]{2}{*}{$\underset{(3)}{\log F_{\mathrm{CIV}}}$} & \multicolumn{2}{|c|}{$f_{\mathrm{BC}} / f_{\mathrm{tot}}$} & \multicolumn{2}{|c|}{$v_{\mathrm{NC}}-v_{\mathrm{BC}}$} & \multirow{2}{*}{$\begin{array}{c}\xi_{\mathrm{NC}} \\
\left(\mathrm{km} \mathrm{s}^{-1}\right) \\
(8)\end{array}$} & \multirow{2}{*}{$\begin{array}{c}\xi_{\mathrm{BC}} \\
\left(\mathrm{km} \mathrm{s}^{-1}\right) \\
(9)\end{array}$} & \multirow[b]{2}{*}{$\begin{array}{c}\text { REFERENCE } \\
(10)\end{array}$} \\
\hline & & & $\begin{array}{c}\text { Si IV } \\
\text { (4) }\end{array}$ & $\underset{(5)}{C \text { IV }}$ & $\begin{array}{l}\text { Si IV } \\
(6)\end{array}$ & $\begin{array}{c}\mathrm{C} \text { IV } \\
(7)\end{array}$ & & & \\
\hline $\mathrm{AU} \mathrm{Mic}$ & 7.55 & 5.28 & $0.56 \pm 0.07$ & $0.43 \pm 0.03$ & $2 \pm 5$ & $-9 \pm 3$ & $15.4 \pm 1.5$ & $96.5 \pm 9.2$ & $1,2,3$ \\
\hline HR $1099 \ldots \ldots$ & 7.40 & 5.84 & $0.55 \pm 0.14$ & $0.66 \pm 0.03$ & (0) & $8 \pm 2$ & $48.5 \pm 4.7$ & $149.7 \pm 2.4$ & 4,5 \\
\hline AB Dor.......... & 6.79 & 5.49 & & $0.49 \pm 0.03$ & 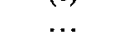 & $6 \pm 4$ & $39.1 \pm 1.2$ & $200.3 \pm 4.8$ & 6,7 \\
\hline 31 Com ........ & 6.22 & 5.57 & $0.39 \pm 0.06$ & $0.62 \pm 0.04$ & $10 \pm 5$ & $9 \pm 4$ & $78.8 \pm 9.7$ & $179.1 \pm 17.0$ & 8 \\
\hline Capella ........ & 5.38 & 5.33 & $0.35 \pm 0.04$ & $0.52 \pm 0.05$ & $19 \pm 4$ & $-4 \pm 4$ & $78.3 \pm 5.8$ & $186.9 \pm 13.4$ & $3,8,9$ \\
\hline Procyon ....... & 5.19 & 4.65 & $<\overline{0.20}$ & $<0.20$ & & $\ldots$ & $45.3 \pm 1.3$ & & 5,8 \\
\hline$\beta$ Cet........... & 5.02 & 3.76 & $0.38 \pm 0.03$ & $0.41 \pm 0.03$ & $-13 \pm 2$ & $-12 \pm 3$ & $42.7 \pm 1.8$ & $109.1 \pm 4.1$ & 8 \\
\hline$\alpha$ Cen B ....... & 4.80 & 3.81 & $0.28+0.05$ & $\ldots$ & $6 \pm 2$ & $\ldots$ & $22.6 \pm 1.2$ & $51.3 \pm 3.6$ & 8 \\
\hline Sun ............ & 4.51 & 3.98 & & . & $\ldots$ & $\ldots$ & $26.0 \pm 5.0$ & & $10,11,12$ \\
\hline$\beta$ Dra $\ldots \ldots \ldots$ & 4.38 & 4.59 & $0.23 \pm 0.05$ & $0.35 \pm 0.04$ & $4 \pm 6$ & $-1 \pm 8$ & $78.3 \pm 6.7$ & $160.0 \pm 20.4$ & 8 \\
\hline$\alpha \operatorname{Cen} A \ldots \ldots$ & 4.06 & 3.73 & $0.25 \pm 0.04$ & & $9 \pm 3$ & $\ldots$ & $27.6 \pm 1.2$ & $65.2 \pm 6.0$ & 8 \\
\hline$\beta$ Gem $\ldots \ldots \ldots$ & 3.16 & 2.76 & $<0.20$ & $<0.20$ & $\ldots$ & $\ldots$ & $48.2 \pm 3.4$ & $\ldots$ & 8 \\
\hline
\end{tabular}

REFERENCES.-Wood et al. 1994; (2) Linsky \& Wood 1994; (3) Wood 1996; (4) Dempsey et al. 1993a; (5) Wood et al. 1996; (6) Hempelmann et al. 1995; (7) Vilhu et al. 1996; (8) Ayres et al. 1995; (9) Linsky et al. 1995; (10) Maggio et al. 1987; (11) Ayres, Marstad, \& Linsky 1981; (12) Dere \& Mason 1993.

the other data points. However, Procyon might not be as discrepant as these figures suggest. The broad component contributions plotted in Figures $12 d-12 f$ are only for the $\mathrm{Si}$ IV and C IV lines. No broad component was detected for Procyon in these lines, but a broad component was detected in the $\mathrm{N} v \lambda 1239$ line. The $\mathrm{N} v$ broad component was found to account for $42 \% \pm 8 \%$ of the total line flux, so if a $\mathrm{N} v$ data point were plotted for Procyon, it would be consistent with the $\mathrm{Si}$ IV and $\mathrm{C}$ rv data points of the other stars. The $\mathrm{N} v$ line is formed at somewhat higher temperatures than the Si IV and C rv lines. Perhaps for Procyon, which is the hottest star in our sample, the broad component phenomenon only becomes evident at higher temperatures in the transition region.

If microflaring is indeed the correct explanation for the broad components, then Figure 12 suggests that the micro-

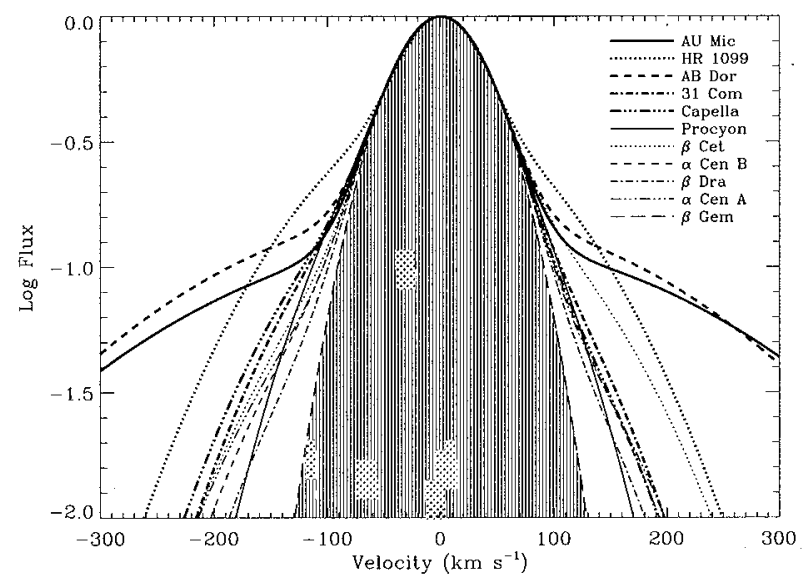

FIG. 11. Comparison of the transition region line profiles of all the stars in our sample, corrected for instrumental and rotational broadening, based on the fits to the data shown in Fig. 10 (except for Procyon-see text). The $\mathrm{C}$ rv profile of AB Dor is also shown, based on the two-Gaussian fit of Vilhu et al. (1996). In order to compare the profile shapes properly, all the lines have been normalized to have the same amplitude (1.0) and FWHM (100 $\left.\mathrm{km} \mathrm{s}^{-1}\right)$, and at the half-maximum location we force the lines to be centered at a velocity of $0 \mathrm{~km} \mathrm{~s}^{-1}$. The profiles have been plotted on a $\log$ flux scale in order to emphasize the line wings. The shaded region indicates what a Gaussian profile would look like when plotted this way. In the figure, the stars are listed in order of decreasing X-ray surface flux. The more active stars clearly tend to have the broader line wings. flaring must be more prevalent on active stars than on inactive stars. It certainly would not be surprising if this were the case. In addition to the microflaring hypothesis, Vilhu et al. (1996) proposed another interpretation for the broad component of AB Dor. They suggested that the broad wing emission could originate in large "slingshot prominences" that extend several stellar radii above the surface of the star and rigidly rotate with the star. Because AB Dor is rotating very rapidly ( $v \sin i=90 \mathrm{~km} \mathrm{~s}^{-1}$ ), emission in these slingshot prominences would be rotationally broadened in a way that could produce the extensive wings observed in C IV. This hypothesis is plausible because $\mathrm{H} \alpha$ observations suggest that prominences this large do indeed exist on $\mathrm{AB}$ Dor (Cameron \& Robinson 1989). It is uncertain, however, whether such structures contain gas hot enough and abundant enough to account for the broad components of the C rv lines.

Figure 11 shows that the $C$ iv lines of $A B$ Dor and $A U$ Mic have very similar shapes. However, it is unlikely that the slingshot prominence interpretation is also applicable for AU Mic, because its rotation rate is only $v \sin i \leq 5.9$ $\mathrm{km} \mathrm{s}^{-1}$ (see Table 1). In order to explain the broad C IV wings of $\mathrm{AU} \mathrm{Mic}$, rigidly rotating prominences would have to extend out to at least 15 stellar radii, which seems improbable. Also, slingshot prominences cannot explain why the transition region line wings of $\beta$ Cet are more extended than those of the similar, but much less active star $\beta \mathrm{Gem}$, because both are slow rotators (see Table 1). Thus, we believe that microflaring is a better interpretation of the broad $\mathrm{C}$ IV line wings, as it seems to be more broadly applicable.

Another attraction of the microflaring interpretation is the possible existence of a well-studied solar analog of the phenomenon, namely transition region explosive events (see, e.g., Dere, Bartoe, \& Brueckner 1989). The GHRS observations of $\alpha$ Cen A provide an excellent opportunity to test the connection between the solar explosive events and the stellar broad components because $\alpha$ Cen A is nearly identical to the Sun in almost all respects, including age (about $5 \times 10^{9} \mathrm{yr}$ ) and spectral type (G2 V). The width of the broad component of $\alpha$ Cen A is $109 \pm 10 \mathrm{~km} \mathrm{~s}^{-1}$, which agrees very well with the line widths typically produced by the solar explosive events. Although the centroids of the 

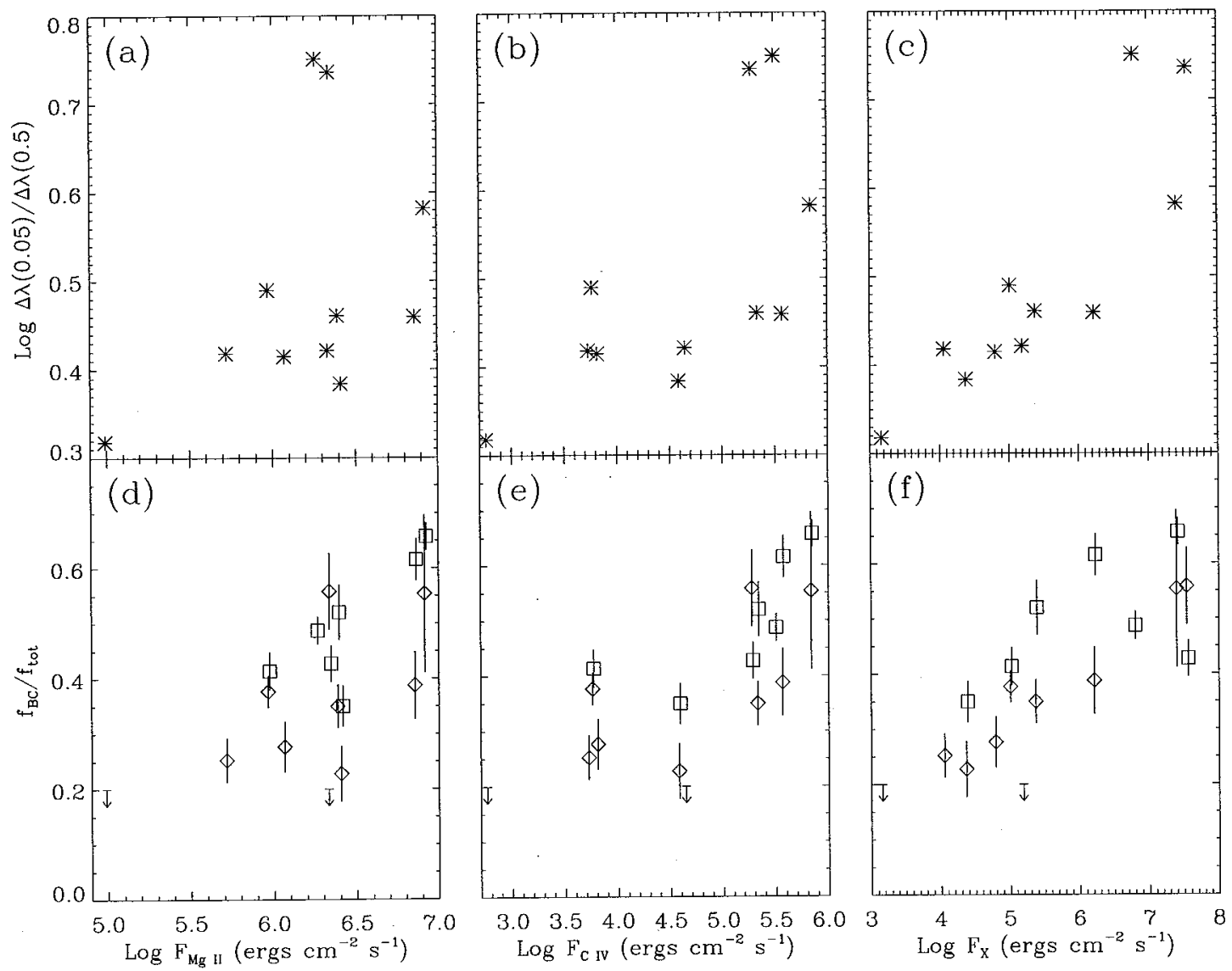

FIG. 12.-Two quantities measuring the prominence of the line wing emission are plotted vs. the $\mathrm{Mg}$ II, $\mathrm{C}$ iv, and X-ray surface fiuxes. The first quantity $[\Delta \lambda(0.05) / \Delta \lambda(0.5)]$ is the width of the line profile at $5 \%$ of the maximum amplitude divided by the FWHM, which is plotted in panels $(a)-(c)$. The second quantity $\left(f_{\mathrm{BC}} / f_{\text {tot }}\right)$ is the flux of the broad component of the two-Gaussian fit divided by the total line flux, which is plotted in panels $(d)-(f)$ for both the $\mathrm{C}$ rV (boxes) and $\mathrm{Si} \mathrm{IV}$ (diamonds) lines.

emission lines produced by individual solar explosive events vary greatly, Dere et al. (1989) found that blueshifted profiles are more common than redshifted profiles, which may explain why the broad component of $\alpha$ Cen $\mathrm{A}$ is blueshifted relative to the narrow component by $9 \pm 3 \mathrm{~km} \mathrm{~s}^{-1}$ (see Table 8). Inspection of columns (6) and (7) of Table 8 reveals a general tendency for the broad components to be blueshifted relative to the narrow components. The only clear examples of redshifted broad components are the Si IV and $\mathrm{C}$ IV lines of $\beta$ Ceti, and possibly the C Iv lines of AU Mic.

One problem with the solar explosive event interpretation of the broad components is that the broad component of $\alpha$ Cen $A$ accounts for $25 \% \pm 4 \%$ of the total line flux, whereas explosive events appear to account for only about $5 \%$ of the Sun's total transition region line flux (Linsky \& Wood 1994). If the Sun were plotted in Figures $12 c$ and $12 d$ with $f_{\mathrm{BC}} / f_{\text {tot }}=0.05$, it would be inconsistent with the general trend suggested by the other data points. There are a number of possible explanations for this problem:

1. The amount of microflaring in the solar transition region is anomalously low.

2. The prevalence of the explosive events in the solar transition region has been underestimated, possibly owing to insufficient spatial and/or time resolution.

3. Stellar transition region explosive events are not responsible for the observed broad wing emission seen in the Si IV and C Iv lines.
4. The line fluxes produced by the stellar explosive events are overestimated by the two-Gaussian fits to the diskaveraged line profiles.

The first of these explanations does not seem very likely, because to our knowledge the Sun is not a particularly unusual star in any other respect. It is difficult for us to assess the plausibility of the second explanation, but Parker (1988) has previously proposed that the explosive events observed by HRTS are only the most energetic of the solar microflares and that most of the microflaring activity on the Sun is as yet undetectable. Hopefully, the recently launched Solar Heliospheric Observatory ( $\mathrm{SOHO}$ ) will shed some light on this issue. If the third explanation is true, that would cast serious doubt on the whole microflaring interpretation of the broad wings, as we are aware of no other solar phenomenon that could be the solar analog of the hypothetical microflaring. The last explanation is quite possible, because even if microflares are the correct explanation for the broad wings of the stellar transition region lines, this does not mean that simple two-Gaussian fits accurately quantify the properties of these microflares.

If either the third or fourth explanations is true, then the nonmicroflaring component of the line profile must contribute more emission to the line wings than our Gaussian narrow components suggest. We believe that this is almost certainly true to some extent for the following reasons. First of all, Dere \& Mason (1993) find that solar-quiet region 
Si IV and C IV line profiles generally have excess emission in the line wings when compared with a Gaussian profile fitted to the emission lines. (However, this might conceivably be due to unresolved microflaring.) Furthermore, even if we disregard explosive events, the widths of Si IV and C IV profiles still vary significantly from place to place on the solar surface. Even if we assume that these profiles are Gaussian, the sum of Gaussians with different widths will still result in a profile with wings extended beyond those of a Gaussian profile. Thus, even in the absence of transition region explosive events, we expect that disk-averaged solar/ stellar line profiles will have some broad wing emission. Wood et al. (1996) demonstrated that a decent fit to the C IV lines of HR 1099 could be achieved without a broad component, if one assumes that a very broad distribution of line widths are being emitted from the stellar surface (see their Fig. 17). We note here, however, that models such as this cannot explain the observed line asymmetries, which are indicated in Table 8 by the velocity differences between the broad and narrow components, seen for HR 1099 and most of the other stars in our sample.

For the particular case of $\alpha$ Cen A, we have performed another two-component fit to the data in which we assume that the narrow component is not simply Gaussian but is instead emitted from a transition region producing Gaussian line profiles with a distribution of widths. We assume a normal distribution of line widths, with the distribution width parameter, $\sigma$, set to be $30 \%$ of the average of the distribution. This assumed distribution roughly mimics the solar case (Dere \& Mason 1993). The resulting fit is depicted in Figure 13. In addition to the two components and their sum (solid lines), we also show a Gaussian profile (dotted line) with the same velocity and flux as the narrow component of the fit and with a width identical to the average of the narrow component's distribution of widths. Comparing the dotted line with the narrow component reveals the

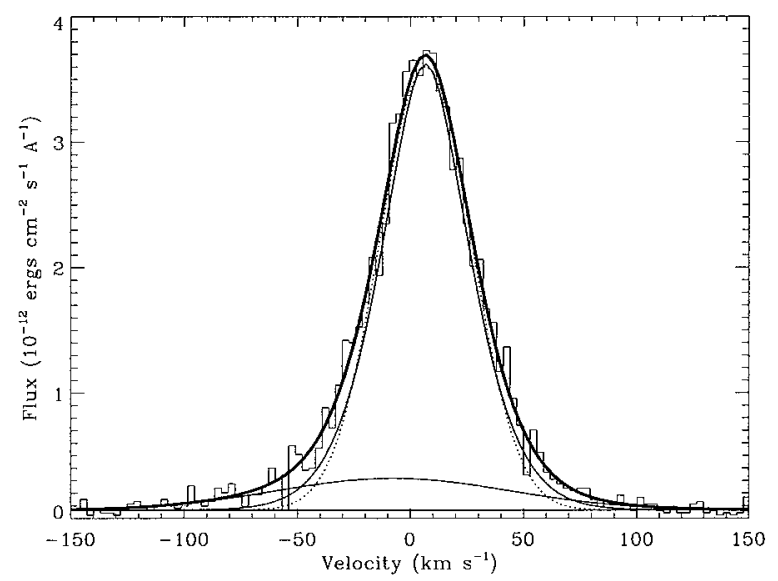

Fig. 13.-Two-component fit to the Si rv $\lambda 1394$ line of $\alpha$ Cen A, where the two components are plotted as thin solid lines and the convolution of their sum with the instrumental profile is shown as a thick solid line. The broad component is simply a Gaussian, but we assume that the narrow component is the sum of many Gaussians with a normal distribution of widths. The width parameter of the distribution, $\sigma$, is assumed to be $30 \%$ of the average of the distribution. This assumption is meant to consider the possibility that not all nonmicroflaring atmospheric regions produce line profiles with exactly the same width. The resulting narrow component is compared with a Gaussian profile (dotted line) that has the same velocity and flux as the narrow component, and has a width equal to the average of the narrow component's distribution of widths. extent to which the assumed line width distribution has broadened the wings of the narrow component. As a consequence, the broad component contribution to the line profile $\left(f_{\mathrm{BC}} / f_{\mathrm{tot}}\right)$ has dropped to $0.15 \pm 0.06$ from a previous value of $0.25 \pm 0.04$ for the fit in Figure $3 a$. This contribution still is larger than the $5 \%$ value believed to be appropriate for the Sun, but this exercise does demonstrate how a simple two-Gaussian fit could significantly overestimate the broad component's flux contribution to the Si IV and C IV lines.

\subsection{Narrow Components and Nonthermal Velocities}

In Figure $14 a$, we plot the transition region nonthermal velocities, as measured by the widths of single-Gaussian fits to the Si IV and C IV lines, versus the stellar surface gravity. The surface gravities were computed from the masses and radii listed in Table 1 . In Figure $14 b$, we plot the nonthermal velocities of the narrow and broad components versus surface gravity. The dashed lines in both panels of Figure 14 indicate the sound speed at a temperature of $10^{5} \mathrm{~K}$. The broad component's nonthermal velocities in all cases are supersonic. This leads us to speculate that the large widths of the broad components are not due to turbulence, per se, because under normal circumstances supersonic turbulence would be degraded rapidly by shock dissipation (see, e.g., Shu 1992).

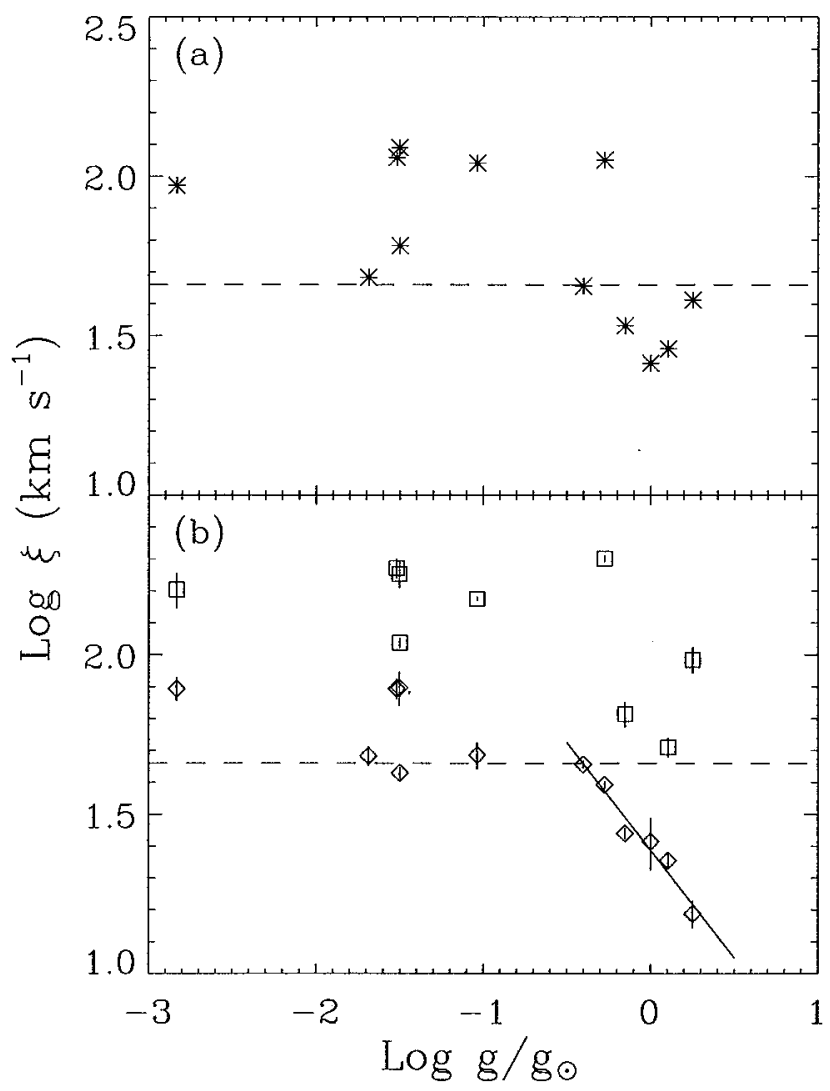

Frg. 14. - (a) Transition region nonthermal velocities, as measured using single-Gaussian fits to the $\mathrm{Si}$ IV and $\mathrm{C}$ IV lines, plotted vs. stellar surface gravity (normalized to the solar value). (b) Transition region nonthermal velocities, as measured by two-Gaussian fits to the Si Iv and C IV lines, plotted vs. surface gravity for both the narrow (diamonds) and broad (boxes) components. The dashed lines indicate the sound speed at $10^{5} \mathrm{~K}$. Also displayed is a linear least-squares fit to the narrow component velocities of the six stars with the highest surface gravities (solid line). 
One of the attractive aspects of the microflaring interpretation of the broad components is that it suggests an alternative explanation for the large line widths. Flares and microflares produce broad emission lines owing to highvelocity jets of plasma ejected from the magnetic reconnection site rather than owing to isotropic turbulence. We do not expect line profiles produced under these conditions to be Gaussian, and in fact most solar explosive events generate $\mathrm{C}$ IV lines that are far from Gaussian. The sum of thousands of such profiles, from different locations on the stellar disk, might result in a profile that is close to Gaussian, but the width of this Gaussian will not be characteristic of the true turbulent velocity.

Of the six low-gravity, evolved stars in our sample, three have narrow components with widths that suggest nonthermal velocities comparable to the sound speed, and three ( $\beta$ Dra, Capella, and $31 \mathrm{Com}$ ) have narrow component nonthermal velocities about $60 \%$ larger than the sound speed (see Fig. 14b). The latter are the same three stars for which we believe that the $\mathrm{C}$ IV and Si IV lines are broadened by opacity effects and/or line blending (see $§ 4)$. Thus, it is likely that the narrow-component nonthermal velocities have been significantly overestimated for these three stars. We believe that our data in Figure $14 b$ are consistent with the narrow component having a constant nonthermal velocity, equal to the sound speed, for stars with surface gravities between $\log g / g_{\odot}=-3.0$ and $\log g / g_{\odot}=-0.4$. For stars with larger gravities, the nonthermal velocity decreases with increasing surface gravity. We have performed an analytical least-squares fit to quantify this correlation for $\log g / g_{\odot}>$ -0.4 (see Fig. 14b), and we find that

$$
\xi_{\mathrm{NC}}=(24 \pm 1)\left(\frac{g}{g_{\odot}}\right)^{-0.68 \pm 0.07} .
$$

Because of the efficiency of shock dissipation in damping supersonic motions, it is not surprising that the $\xi_{\mathrm{NC}}$ versus $g$ relation flattens as soon as $\xi_{\mathrm{NC}}$ reaches the sound speed.

Note that this apparently tight correlation between $\xi_{\mathrm{NC}}$ and $g$ is not evident in Figure 14a. The solar nonthermal velocity plotted in Figure 14 is the result of spatially resolved $\mathrm{C}$ IV and $\mathrm{Si}$ IV observations of solar quiet and active regions (Dere \& Mason 1993). In Figure 14a the solar nonthermal velocity is lower than the stellar velocities measured from the disk-averaged line profiles, but in Figure $14 b$ the solar velocity agrees very well with the correlation between $\xi_{\mathrm{NC}}$ and $g / g_{\odot}$. The existence of the Figure $14 b$ correlation, and the agreement of this correlation with the solar value, represents a possible validation of our use of two-Gaussian fits to quantify transition region line profiles.

The significance of the narrow-component velocities is that they represent a substantial amount of kinetic energy, possibly enough to heat the entire transition region. Are these nonthermal velocities due to isotropic turbulence, or are they caused by the passage of waves through the transition region? The flux in a propagating wave can be written as (see, e.g., Brown \& Jordan 1981)

$$
F_{\text {wave }}=\rho\left\langle V_{T}^{2}\right\rangle V_{\text {prop }},
$$

where $\rho$ is the density, $V_{\text {prop }}$ is the propagation velocity, and $\left\langle V_{T}^{2}\right\rangle$ is the root mean square velocity $\left(\left\langle V_{T}^{2}\right\rangle=3 / 2 \xi_{\mathrm{NC}}^{2}\right)$. If the wave is a sound wave, $V_{\text {prop }}$ is simply the sound speed, which is about $46 \mathrm{~km} \mathrm{~s}^{-1}$ at the line formation temperature of C IV. For a plasma with solar abundances, hydrogen accounts for about $70 \%$ of the total mass, and at transition region temperatures $n_{\mathrm{H}} \approx 0.8 n_{e}$ (McWhirter, Thoneman, \& Wilson 1975). Thus, the density $\rho$ can be related to the electron density, $n_{e}$, by the expression $\rho=1.14 m_{p} n_{e}$, where $m_{p}$ is the proton mass. Noting that the electron pressure at $10^{5} \mathrm{~K}$ (in units of $\mathrm{cm}^{-3} \mathrm{~K}$ ) is $P_{e}=10^{5} n_{e}$, we can then rewrite equation (3) as

$$
F_{S}=79 m_{p} P_{e} \xi_{\mathrm{NC}}^{2}
$$

If the wave is an Alfvén wave, $V_{\text {prop }}$ is the Alfvén velocity, and equation (3) becomes

$$
F_{\mathrm{A}}=1.4 \times 10^{-3}\left(m_{p} P_{e}\right)^{1 / 2} \xi_{\mathrm{NC}}^{2} B
$$

In Kolmogorov turbulent dissipation, energy cascades from the largest turbulent eddies to progressively smaller and smaller eddies until the bulk kinetic energy finally is converted to thermal energy through viscosity or Joule heating. The heating rate for the conversion of turbulent energy to thermal energy is (Shu 1992; Narain \& Ulmschneider 1990, 1996)

$$
\epsilon_{t}=\frac{\rho\left\langle V_{T}^{2}\right\rangle^{3 / 2}}{L},
$$

where $L$ is the scale size of the largest turbulent eddies (i.e., the scale size in which the turbulent energy first appears). A surface flux for turbulent energy can be defined as $F_{\text {turb }}=$ $\epsilon_{t} h$, where $h$ is the thickness of the emission layer. We could estimate a value for $h$ from plane-parallel atmospheric models of the Sun and other stars $(h \sim 10 \mathrm{~km}$ for the solar transition region lines; Nicolas et al. 1979), but it is very hard to know what value we should assume for $L$. Thus, we simply assume $L=h$, which corresponds to the situation in which the largest turbulent eddies span the width of the entire emission layer. This is a crude estimate, but it may not be unreasonable for the very thin transition region. Replacing $\rho$ with $1.14 m_{p} n_{e},\left\langle V_{T}^{2}\right\rangle$ with $3 / 2 \xi_{\mathrm{NC}}^{2}$, and $n_{e}$ with $10^{-5} P_{e}$, we then have

$$
F_{\text {turb }}=2.1 \times 10^{-5} m_{p} P_{e} \xi_{\mathrm{NC}}^{3} .
$$

We now wish to compare $F_{S}, F_{\mathrm{A}}$, and $F_{\text {turb }}$ with $F_{\text {rad }}$, the radiative flux of the narrow component. The calculations of McWhirter et al. (1975) show that

$$
F_{\mathrm{rad}}=2.4 \times 10^{-22} E_{m}
$$

at the line formation temperature of $\mathrm{C} \mathrm{IV}, T=10^{5} \mathrm{~K}$, where $E_{m}$ is the emission measure. (For a homogeneous planeparallel atmosphere, $E_{m}=n_{e}^{2} h$.) The observed C rv surface flux $F_{\mathrm{CIV}}$, which is computed assuming the $\mathrm{C}$ IV flux is emitted uniformly over the stellar surface, is related to the emission measure by the equation (Brown et al. 1984)

$$
F_{\mathrm{CIV}}=1.65 \times 10^{-23} E_{m} a,
$$

where $a$ is the surface filling factor $(0<a<1)$. Combining relations ( 8 ) and (9), we then have

$$
a F_{\mathrm{rad}}=14.5 F_{\mathrm{C} \mathrm{IV}}\left(1-f_{\mathrm{BC}} / f_{\mathrm{tot}}\right),
$$

where the factor $\left(1-f_{\mathrm{BC}} / f_{\text {tot }}\right)$ ensures that the C IV surface flux considered is for the narrow component only.

Waves propagating through the transition region may deposit much of their energy in the corona as well as in the transition region, so we also wish to compare $F_{S}$ and $F_{\mathrm{A}}$ with the coronal radiative flux, $F_{\text {cor }}$, computed from the $F_{\mathrm{x}}$ 
values in Table 8. Based on the plasma models of Mewe, Gronenschild, \& van den Oord (1985) and Mewe, Lemen, \& van den Oord (1986),

$$
F_{\text {cor }}= \begin{cases}6 \times 10^{-23} E_{m} & \text { for } \log T_{\text {cor }}=6.0 \\ 2 \times 10^{-23} E_{m} & \text { for } \log T_{\text {cor }}=7.0,\end{cases}
$$

where $T_{\text {cor }}$ is the coronal temperature. For X-ray fluxes measured in the Einstein IPC or ROSAT PSPC bandpasses, like those listed in Table 8,

$$
F_{\mathrm{X}}=1.4 \times 10^{-23} E_{m} a_{\text {cor }} \eta
$$

for both $\log T_{\text {cor }} \approx 6.0$ and $\log T_{\text {cor }} \approx 7.0$, where $a_{\text {cor }}$ is the surface filling factor for coronal emission and $\eta$ is a geometrical factor between 0.5 and 1.0. If the coronal emission is emitted close to the stellar surface, half the radiation from the plasma is emitted into the star rather than into interstellar space, and $\eta=0.5$. If the corona is very extended, however, and the star intercepts very little of the coronal emission, then $\eta \approx 1.0$. For the Sun, $\eta$ is much closer to 0.5 than to 1.0. Although this may not be the case for the other stars, we simply assume $\eta=0.5$ and combine equations (11) and (12) to find that

$$
a_{\mathrm{cor}} F_{\text {cor }}= \begin{cases}8.6 F_{\mathrm{X}} & \text { for } \log T_{\text {cor }}=6.0 \\ 2.9 F_{\mathrm{X}} & \text { for } \log T_{\text {cor }}=7.0\end{cases}
$$

Electron densities have been estimated from GHRS spectra for seven of the stars in our sample using different diagnostics (Maran et al. 1994; Wood et al. 1996; Linsky et al. 1995; Wood 1996; Dere \& Mason 1993). Since $P_{e}$ should be roughly constant in the transition region (see, e.g., Jordan et al. 1987), electron pressures computed from these densities should apply at $T=10^{5} \mathrm{~K}$. Note that the lines used as density diagnostics have not been separated into narrow and broad components (the $\mathrm{O}$ IV] lines, for example, are too weak and/or too blended; see, e.g., Linsky et al. 1995), and it is hard to judge how accurate densities computed from total line fluxes and ratios are in the presence of substantial inhomogeneity (see Judge, Hubeny, \& Brown 1997).

Nevertheless, the electron pressures are listed in Table 9, along with values for $a F_{\text {rad }}, a_{\mathrm{cor}} F_{\text {cor }}, F_{S}, F_{\mathrm{A}} / B$, and $F_{\text {turb }}$ computed using equations (10), (13), (4), (5), and (7), respectively. In computing these quantities, we use the values of $F_{\mathrm{CIV}}, F_{\mathrm{X}}, f_{\mathrm{BC}} / f_{\text {tot }}$, and $\xi_{\mathrm{NC}}$ given in Table 8 . We use the C IV values listed for $f_{\mathrm{BC}} / f_{\mathrm{tot}}$, with the exception of $\alpha$ Cen A and B for which we are forced to use the Si IV values. For Capella, we assume that $\xi_{\mathrm{NC}}$ is equal to the sound speed $\left(46 \mathrm{~km} \mathrm{~s}^{-1}\right)$ rather than the velocity listed in Table 8, which we believe is an overestimate. We have to assume a value for $T_{\text {cor }}$ to

\begin{tabular}{|c|c|c|c|c|c|c|}
\hline \multirow[b]{2}{*}{ StAR } & \multirow{2}{*}{$\underset{\left(\mathrm{cm}^{-3} \stackrel{\operatorname{Kog}}{\mathrm{K}}\right)}{\log \mathbf{P}_{e}}$} & \multicolumn{5}{|c|}{ FLuXXes $\left(10^{5} \mathrm{ergs}^{-2} \mathrm{~s}^{-1}\right)$} \\
\hline & & $a F_{\mathrm{rad}}$ & $a_{\text {cor }} F_{\text {cor }}$ & $F_{S}$ & $F_{\mathrm{A}} / B$ & $F_{\text {turb }}$ \\
\hline AU Mic & 16.03 & 16 & 1000 & 33 & 4.4 & 14 \\
\hline HR $1099 \ldots$ & 14. & 34 & 730 & 30 & 13 & 39 \\
\hline Capella .. & 15.04 & 15 & 7.0 & 30 & 13 & 36 \\
\hline Procyon ... & $<14.92$ & 6.5 & 14 & $<23$ & $<11$ & $<27$ \\
\hline$\alpha \operatorname{Cen}$ B ... & 14.64 & 0.67 & 5.6 & 3.0 & 2.0 & 1.8 \\
\hline Sun & 15.00 & 1.4 & 2.9 & 8.9 & 3.9 & 6.2 \\
\hline$\alpha$ Cen A ... & 14.79 & 0.58 & 1.0 & 6.2 & 3.4 & 4.6 \\
\hline
\end{tabular}

TABLE 9

Comparing Radiative, Wave, and Turbulent Fluxes estimate $a_{\text {cor }} F_{\text {cor }}$. For AU Mic, HR 1099, and Capella, we assume $\log T_{\text {cor }}=7.0$ (Maran et al. 1994; Dempsey et al. $1993 \mathrm{~b}$ ), and for the less active stars, we assume $\log T_{\text {cor }}=$ 6.0 (Mewe et al. 1995; Schrijver et al. 1995).

Based on Table 9, for AU Mic and HR 1099 acoustic waves might barely be able to account for the narrow component radiative fluxes observed from the $\mathrm{C}$ IV emission layer, but they clearly cannot be responsible for even a significant fraction of the observed coronal emission. Thus, for AU Mic and HR 1099, acoustic waves are not a very promising source of heating. The situation is more ambiguous for the five less active stars listed in Table 9. For these stars, the estimated acoustic wave fluxes are large enough to account for much of the transition region and coronal radiation. Jordan et al. (1987) obtained similar results in their analysis of five main-sequence stars, including $\alpha$ Cen $\mathrm{A}$ and B. However, they pointed out that while acoustic waves may conceivably be able to account for the radiative losses from the stellar coronae, they cannot also account for expected conductive losses. More detailed analyses of the solar chromosphere and transition region using spatial- and time-resolved spectra have also cast doubt on the viability of the acoustic wave heating hypothesis (see, e.g., Athay \& White 1978; Bruner 1978). We note that the $F_{S}$ values in Table 9 may actually be significantly lower than the $F_{\text {rad }}$ and $F_{\text {cor }}$ values for all the stars listed in the table if the surface filling factors, $a$ and $a_{\mathrm{cor}}$, are significantly less than 1, which the solar example suggests is probably the case for the less active stars (especially for $a_{\text {cor }}$ ).

Another argument against the acoustic wave heating hypothesis is that we believe the magnetic field must play some role in increasing the narrow-component fluxes for more active stars. Although the narrow-component contribution to the $\mathrm{C}$ IV and Si IV lines decreases with increasing activity, the surface flux of the narrow component is still much larger for the active stars than for the inactive stars. For example, the narrow-component $\mathrm{C}$ IV surface flux of $\beta$ Cet is 6 times larger than the total $\mathrm{C}$ IV surface flux of $\beta$ $\mathrm{Gem}$. It is difficult to explain this behavior without some type of magnetic heating process. Similar arguments against acoustic wave heating have been made in the past. The discovery that coronal activity is strongly correlated with stellar rotation clearly points toward a magnetic heating mechanism (see, e.g., Pallavicini et al. 1981).

Alfvén waves are a more plausible candidate for the narrow-component heating mechanism than acoustic waves. Table 9 demonstrates that it takes a magnetic field of only a few gauss for $F_{\mathrm{A}}$ to exceed $F_{S}$, and we can increase $B$ even further in order to make $F_{\mathrm{A}}$ comparable to $F_{\mathrm{rad}}$ and $F_{\text {cor }}$. For the Sun, a magnetic field of $B \approx 10 \mathrm{G}$ may be appropriate (Bruner 1978). For very active stars like AU Mic, photospheric magnetic fields as high as $B \approx 4000 \mathrm{G}$ have been found to cover a large fraction of the stellar surface (Johns-Krull \& Valenti 1996). Jordan et al. (1987) also concluded that Alfvén waves are a viable source of transition region and coronal heating, based on calculations similar to ours.

There are many other types of MHD waves besides Alfvén waves that have been proposed as potential heating sources (see reviews by Narain \& Ulmschneider 1990, 1996). In stellar atmospheres in which the Alfvén speed can be expected to exceed the sound speed, slow-mode MHD waves and longitudinal wave modes in flux tubes do not propagate faster than the sound speed and therefore cannot 
carry more flux than acoustic waves. Also, increasing the magnetic field does not increase the flux. Thus, all of the arguments presented above against acoustic wave heating also apply to heating by slow-mode and longitudinal MHD waves. In contrast, fast-mode MHD waves propagate at or above the Alfvén speed, meaning that these waves, like the Alfvén waves, are viable sources of transition region and coronal heating.

A potential problem with wave-heating mechanisms is that one must be able to explain how the wave energy is dissipated. The usual picture is that after the waves are generated by subphotospheric convection, they travel upward through the atmosphere and eventually steepen into shock waves, thereby converting their energy to thermal energy through shock dissipation. However, the details of how this occurs have not yet been completely worked out.

In contrast, if the narrow component nonthermal velocities are interpreted as turbulent velocities, conversion of the turbulent energy to thermal energy is not difficult to explain. One would expect turbulence to be naturally dissipated through a Kolmogorov cascade process. The values of $F_{\text {turb }}$ in Table 9 suggest turbulent heating may indeed be a viable heating mechanism, since as long as $a$ is not too small, $F_{\text {turb }} \approx F_{\text {rad }}$. There is no point in comparing $F_{\text {turb }}$ with $F_{\text {cor }}$, because turbulent energy should be dissipated locally. Thus, for the turbulent dissipation interpretation to work, we have to show only that $F_{\text {turb }} \approx F_{\text {rad }}$, in contrast to the wave fluxes, for which we want $F_{\text {wave }} \gtrsim F_{\text {rad }}+F_{\text {cor }}$. Although $F_{\text {turb }}$ does appear to be comparable to $F_{\text {rad }}$, we caution that uncertainties in the calculation are high, as equation (7) is more of an order-of-magnitude kind of equation than equations (4) and (5). In a similar calculation, Nicolas et al. (1979) also found that turbulent dissipation may be able to account for the transition region heating on the Sun, assuming once again that $a$ is not too small. We now present another argument in favor of the turbulent heating hypothesis.

Jordan et al. (1987) found empirically that

$$
F_{\mathrm{Mg} \mathrm{II}} \propto P_{e}^{3 / 5} g^{-1}
$$

for main-sequence stars. Combining this relation with the relation

$$
F_{\mathrm{CIV}} \propto F_{\mathrm{Mg} \mathrm{II}}^{1.77 \pm 0.12}
$$

from Ayres et al. (1995), we find that

$$
F_{\mathrm{CIV}} \propto P_{e}^{1.06 \pm 0.07} g^{-1.77 \pm 0.12} .
$$

Since equation (2) provides us with an empirical relation between $\xi_{\mathrm{NC}}$ and $g$, we can express equation (7) in terms of $P_{e}$ and $g$. If we then equate $F_{\text {turb }}$ and $F_{\text {rad }}$ in equations (7) and (10), we can derive an expression analogous to equation (16) and test the $F_{\text {rad }}=F_{\text {turb }}$ assumption. Since equation (16) originates from analyses that assume homogeneity and spherical symmetry, for the purposes of this comparison we assume $a=1$ and $\left(1-f_{\mathrm{BC}} / f_{\mathrm{tot}}\right)=1$. We then have

$$
F_{\mathrm{CIV}} \propto P_{e} g^{-2.04 \pm 0.21} \text {. }
$$

Equations (16) and (17) agree very well, which provides support for the $F_{\text {rad }}=F_{\text {turb }}$ assumption. It is possible that the narrow-component heating mechanism, whatever it may be, deposits its energy into the transition region through turbulence, and the transition region is then heated by the dissipation of this turbulence. We are not sure what the source of the turbulent energy is, but as mentioned above, we believe that magnetic activity must be involved. Considering the vast literature that exists concerning possible coronal heating mechanisms, it should come as no surprise that there are many theories that are compatible with the requirements that the narrow-component heating mechanism be connected with magnetic activity and that the energy first be deposited into the upper atmosphere in the form of turbulence. For example, Hollweg (1984) proposed that Alfvén waves are dissipated in the corona through Kolmogorov turbulence. Also, Gómez \& Ferro Fontán (1988) proposed that MHD turbulence can be produced by motions of magnetic loop footpoints induced by subphotospheric convection.

The empirical $\xi_{\mathrm{NC}} \propto g^{-0.68}$ relation that we have observed may be an important diagnostic for the narrow component heating mechanism. In the turbulent dissipation scenario, it is unclear why this relation should exist. Any proposal for the source of the turbulent energy should seek to explain the apparent gravity dependence. In the waveheating scenario, the gravity dependence can be understood through the probable source of the wavessubphotospheric convection, because the convective energy should be dependent on the surface gravity (see, e.g., Stein 1981; Ulmschneider \& Stein 1982).

\section{SUMMARY}

Although the main intent of this paper is to provide a global comparison of the transition region line profiles of late-type stars, for a few stars we do present new results specific to those stars:

1. We find that the emission-line redshifts of $\alpha$ Cen A and B increase with line formation temperature up to $\log T=5.2$. These redshifts are almost identical to those previously observed for the Sun and Procyon, although the redshifts of $\alpha$ Cen B are about $4 \mathrm{~km} \mathrm{~s}^{-1}$ larger than the redshifts of the other three stars. Above $\log T=5.2$, the redshifts decrease dramatically for the Sun, $\alpha$ Cen A, and $\alpha$ Cen B, but for Procyon, they continue to increase.

2. Using the $\mathrm{O} \mathrm{IV}]$ lines, we derive electron densities at $\log T=5.14$ of $\log n_{e}=9.65 \pm 0.20$ and $\log n_{e}=9.50$ \pm 0.30 for $\alpha$ Cen A and $\alpha$ Cen B, respectively, corresponding to pressures (in units of $\mathrm{cm}^{-3} \mathrm{~K}$ ) of $\log P_{e}=14.79$ \pm 0.20 and $\log P_{e}=14.64 \pm 0.30$. These pressures are consistent with coronal pressures measured from EUVE data, contrary to what has been found for Capella and Procyon.

3. GHRS observations of the $\mathrm{C}$ IV lines of $\beta$ Dra obtained in three different years (1992, 1993, and 1995) show substantial line profile variability. The reason for this variability in uncertain, but rotational modulation is a possibility.

By comparing the Si IV and C IV profiles of many stars, we have conclusively shown that broad line wings are manifestations of stellar activity, as the prominence of these wings is clearly correlated with both the $\mathrm{C}$ IV and X-ray surface fluxes. We have tried to quantify the generally nonGaussian Si Iv and C Iv profiles with two-Gaussian fits. We believe that microflating is the best interpretation for the broad components of these two-Gaussian fits, for the following reasons:

1. The very large widths of the broad components suggest supersonic velocities. We are reluctant to interpret 
these widths as being due to supersonic turbulence, because supersonic turbulence cannot exist for long due to shock dissipation. By associating the broad components with microflaring, we can explain the broad lines as being due to jets of plasma expelled at high velocities from magnetic reconnection sites.

2. If microflares are in fact common in stellar transition regions, it would not be surprising if they were especially prevalent in the transition regions of very active stars. Thus, the correlation we observe between the broad components and stellar activity is consistent with this interpretation.

3. Solar transition region explosive events, which can be considered a form of microflaring, might be the solar analog for the broad components. For the solar-like star $\alpha$ Cen A, the broad component has a width of $109 \pm 10 \mathrm{~km} \mathrm{~s}^{-1}$ and is blueshifted relative to the narrow component by $9 \pm 3$ $\mathrm{km} \mathrm{s}^{-1}$. Both of these properties are consistent with the average properties of the solar explosive events. However, for $\alpha$ Cen $\mathrm{A}$, the broad component accounts for about $25 \% \pm 4 \%$ of the total line emission, while it is currently thought that the explosive events account for only about $5 \%$ of the solar Si IV and C IV emission. Perhaps twoGaussian fits overestimate the broad-component contribution to the line flux and/or perhaps the explosive event contribution to the Sun's transition region emission has been underestimated due to insufficient spatial and time resolution.

4. In addition to the solar explosive events and the broad components, other evidence supports the presence of continuous microflaring in the atmospheres of cool stars. For example, very active stars typically have coronal temperatures above $10^{7} \mathrm{~K}$, but on the Sun temperatures this high are seen only during flares (see, e.g., Haisch, Strong, \& Rodonò 1991). Observed correlations between microwave and X-ray emission are similar for both active stars and solar flares, which is also suggestive of microflaring (Güdel 1994; Benz \& Güdel 1994).

Finally, we analyze the properties of the narrow components of the two-Gaussian fits and discuss possible heating mechanisms for the narrow component emission. Our main findings are as follows:
1. When we convert the widths of the narrow components to nonthermal velocities $\left(\xi_{\mathrm{NC}}\right)$, we find that the resulting velocities are correlated with stellar surface gravity (g). For the low-gravity evolved stars, our data are consistent with constant $\xi_{\mathrm{NC}}$ equal to the sound speed $(\approx 46 \mathrm{~km}$ $\left.\mathrm{s}^{-1}\right)$. For the high-gravity main-sequence stars, $\xi_{\mathrm{NC}}$ is less than the sound speed and $\xi_{\mathrm{NC}} \propto g^{-0.68}$.

2. Using the empirical relation between $\xi_{\mathrm{NC}}$ and $g$, we can reproduce scaling laws computed for main-sequence stars by Jordan et al. (1987) from atmospheric models based on $I U E$ data, if we assume that the radiative flux is equal to the turbulent flux. This suggests that the narrow components may be associated with heating through turbulent dissipation. Order-of-magnitude calculations suggest that the turbulent and radiative fluxes are indeed roughly equal for the seven stars in our sample that have measured transition region densities, as long as the surface filling factor of the transition region emission is not too low.

3. If the nonthermal velocities, $\xi_{\mathrm{NC}}$, are due to motions induced by Alfvén waves rather than turbulence, it is quite possible that the waves carry enough flux to account for the narrow-component emission and much of the coronal emission. Thus, Alfvén wave dissipation is another viable heating mechanism.

4. Results in this paper and in previous work suggest that acoustic waves are not a likely source of the narrowcomponent emission. For one thing, our data demonstrate that stars with different activity levels that are otherwise very similar can have significantly different narrow component fluxes, which suggests that the heating mechanism is probably associated with magnetic activity.

An important goal for future work is to pursue these issues, particularly for the Sun now that SOHO is available for high-resolution spectroscopic studies of our nearby star.

We would like to thank $P$. Ulmschneider for many useful comments on the manuscript. This work is supported by NASA grant S-56460-D to the National Institute of Standards and Technology.

\section{REFERENCES}

Achour, H., Brekke, P., Kjeldseth-Moe, O., \& Maltby, P. 1995, ApJ, 453, 945

Arnaud, M., \& Rothenflug, R. 1985, A\&AS, 60, 425

The Astronomical Almanac. 1996 (Washington: US Government Printing Office)

Athay, R. G., \& White, O. R. 1978, ApJ, 226, 1135

Ayres, T. R., et al. 1995, ApJS, 96, 223

Ayres, T. R., Jensen, E., \& Engvold, O. 1988, ApJS, 66, 51

Ayres, T. R., Marstad, N. C., \& Linsky, J. L. 1981, ApJ, 247, 545

Baliunas, S. L., et al. 1995, ApJ, 438, 269

Beavers, W. I. \& Eitter, J. J. 1986, ApJS, 62,147

Bell, R. A., \& Gustafsson, B. 1978, A\&AS, 34, 229

Benz, A. O., \& Güdel, M. 1994, A\&A, 285, 621

Bevington, P. R., \& Robinson, D. K. 1992, Data Reduction and Error Analysis for the Physical Sciences (New York: McGraw-Hill)

Blackwell, D. E., Petford, A. D., Arribas, S., Haddock, D. J., \& Selby, M. J. 1990, A\&A, 232, 396

Bopp, B. W. \& Fekel, F. 1977, AJ, 82, 490

Brandt, J. C., et al. 1994, PASP, 106, 890

Brekke, P. 1993, ApJ, 408, 735

Brown, A., \& Jordan, C. 1981, MNRAS, 196, 757

Brown, A., Jordan, C., Stencel, R. E., Linsky, J. L., \& Ayres, T. R. 1984, ApJ, 283, 731

Brown, C. M. 1980, A\&A, 88, 273

Bruner, E. C., Jr. 1978, ApJ, 226, 1140

Cameron, A. C., \& Robinson, R. D. 1989, MNRAS, 238, 657
Char, S., Foing, B. H., Beckman, J., García López, R. J., \& Rebolo, R. 1993, A\&A, 276, 78

Cook, J. W., Keenan, F. P., Dufton, P. L., Kingston, A. E., Pradhan, A. K., Zhang, H. L., Doyle, J. G., \& Hayes, M. E. 1995, ApJ, 444, 936

Demarque, P., Guenther, D. B., \& van Altena, W. F. 1986, ApJ, 300, 773

Dempsey, R. C., Linsky, J. L., Fleming, T. A., \& Schmitt, J. H. M. M. 1993a, ApJS, 86, 599

Dempsey, R. C., Linsky, J. L., Schmitt, J. H. M. M., \& Fleming, T. A. 1993b, ApJ, 413, 333

Dere, K. P., Bartoe, J. -D. F., \& Brueckner, G. E. 1989, Sol. Phys., 123, 41

Dere, K. P., \& Mason, H. E. 1993, Sol. Phys., 144, 217

Doschek, G. A., Feldman, U., \& Bohlin, J.D. 1976, ApJ, 205, L177

Drake, J. J., \& Smith, G. 1991, MNRAS, 250, 89

Dupree, A. K., Brickhouse, N. S., Doschek, G. A., Green, J. C., \& Raymond, J. C. 1993, ApJ, 418, L41

Edlén, B. 1941, Ark. Mat. Astr. Och. Fys., 28B, No. 1

Eriksson, K., Linsky, J. L., \& Simon, T. 1983, ApJ, 272, 665

Fekel, F. C. 1983, ApJ, 268, 274

Flower, P. J. 1977, A\&A, 54, 31

Gilliland, R. L. 1994, GHRS Instrument Science Report 063 (Baltimore: Space Telescope Science Institute)

Gliese, W., \& Jahreiss, H. 1991, Third Catalogue of Nearby Stars (Veröffentlichungen des Astronomishen Rechen-Instituts, University of Heidelberg)

Gómez, D., \& Ferro Fontán, C. 1988, Sol. Phys., 116, 33

Gray, D. F. 1982a, ApJ, 258, 201 
Gray, D. F. 1982b, ApJ, 262, 682

Güdel, M. 1994, ApJS, 90, 743

Guirado, J. C. et al. 1996, in ASP Conf. Proc. 93, Radio Emission from the Stars and the Sun, ed. A. R. Taylor \& J. M. Paredes (San Francisco: ASP), 318

Haisch, B., Strong, K. T., \& Rodonò, M. 1991, ARA\&A, 29, 275

Hallam, K. L. Altner, B., \& Endal, A. S. 1991, ApJ, 372, 610

Hassler, D. M., Rottman, G. J., \& Orrall, F. Q. 1991, ApJ, 372, 710

Heap, S. R., et al. 1995, PASP, 107, 871

Heintz, W.D. 1982, Observatory, 102, 42

Hempelmann, A., Schmitt, J. H. M. M., Schultz, M., Rüdiger, G., \& Stępień, K. 1995, A\&A, 294, 515

Henry, T. J., \& McCarthy, D. W., Jr. 1993, AJ, 106, 773

Hirshfeld, A., Sinnott, R. W., \& Ochsenbein, R. 1991, Sky Catalogue 2000.0, Vol. 1 (Cambridge: Cambridge Univ. Press)

Hoffleit, D., \& Jaschek, C. 1982, The Bright Star Catalogue (New Haven: Yale Univ. Press)

Hollweg, J. V. 1984, ApJ, 277, 392

Hummel, C. A., Armstrong, J. T., Quirrenbach, A., Buscher, D. F., Mozurkewich, D. Elias, N. M., II, \& Wilson, R. E. 1994, AJ, 107, 1859

Iben, I., Jr. 1967, ApJ, 147, 650

Irwin, A. W., Fletcher, J. M., Yang, S. L. S., Walker, G. A. H., \& Goodenough, C. 1992, PASP, 104, 489

Johns-Krull, C. M., \& Valenti, J. A. 1996, ApJ, 459, L95

Jordan, C., Ayres, T. R., Brown, A., Linsky, J. L., \& Simon, T. 1987, MNRAS, 225, 903

Judge, P. G., Hubeny, V., \& Brown, J. C. 1997, ApJ, 475, 275

Kelly, R. L. 1987, J. Phys. Chem. Ref. Data, 16, Suppl. No. 1

Linsky, J. L., et al. 1993, ApJ, 402, 694

Linsky, J. L., \& Wood, B. E. 1994, ApJ, 430, 342

1996, ApJ, 463, 254

Linsky, J. L., Wood, B. E., Judge, P., Brown, A., Andrulis, C., \& Ayres, T. R. 1995, ApJ, 442, 381

Luck, R. E. 1982, ApJ, 256, 177

Lydon, T. J., Fox, P. A., \& Sofia, S. 1993, ApJ, 413, 390

Maggio, A., Sciortino, S., Vaiana, G. S., Majer, P., Bookbinder, J., Golub, L. Harnden, F. R., Jr., \& Rosner, R. 1987, ApJ, 315, 687

Maran, S. P., et al. 1994, ApJ, 421, 800

McWhirter, R. W. P., Thoneman, P. C., \& Wilson, R. 1975, A\&A, 40, 63

Mewe, R., Gronenschild, E. H. B. M., \& van den Oord, G. H. J. 1985, A\&AS, 62, 197

Mewe, R., Kaastra, J. S., Schrijver, C. J., van den Oord, G. H. J., \& Alkemade, F. J. M. 1995, A\&A, 296, 477

Mewe, R., Lemen, J. R., \& van den Oord, G. H. J. 1986, A\&AS, 65, 511

Narain, U., \& Ulmschneider, P. 1990, Space Sci. Rev., 54, 377

. 1996, Space Sci. Rev., 75, 453
Nicolas, K. R., Bartoe, J.-D. F., Brueckner, G. E., \& VanHoosier, M. E. 1979, ApJ, 233, 741

Noels, A., Grevesse, N., Magain, P., Neuforge, C., Baglin, A., \& Lebreton, Y. 1991, A\&A, 247, 91

Oranje, B. J., Zwaan, C., \& Middlekoop, F. 1982, A\&A, 110, 30

Pallavicini, R., Golub, L., Rosner, R., Vaiana, G. S., Ayres, T. R., \& Linsky, J. L. 1981, ApJ, 248, 279

Parker, E. N. 1988, ApJ, 330, 474

Pettersen, B. R. 1980, A\&A, 82, 53

Rucinski, S. M. 1985, MNRAS, 215, 591

Rutten, R. G. M., Schrijver, C. J., Lemmens, A. F. P., \& Zwaan, C. 1991, A\&Á, 252, 203

Sandlin, G. D., Bartoe, J.-D. F., Brueckner, G. E., Tousey, R., \& Van Hoosier, M. E. 1986, ApJS, 61, 801

Schmitt, J. H. M. M., Haisch, B. M., \& Drake, J. J. 1994, Science, 265, 1420

Schrijver, C. J. 1987, A\&A, 172, 111

Schrijver, C. J., Mewe, R., van den Oord, G. H. J., \& Kaastra, J. S. 1995, A\&A, 302,438

Shu, F. H. 1992, The Physics of Astrophysics, Vol. 2 (Mill Valley: University Science Books)

Soderblom, D. R., Sherbert, L. E., \& Hulbert, S. J. 1993, GHRS Instrument Science Report 053 (Baltimore: Space Telescope Science Institute)

Steffen, M. 1985, A\&AS, 59, 403

Stein, R. F. 1981, ApJ, 246, 966

Strassmeier, K. G., \& Fekel, F. C. 1990, A\&A, 230, 389

Strassmeier, K. G., Fekel, F. C., Bopp, B. W., Dempsey, R. C., \& Henry, G. W. 1990, ApJS, 72, 191

Ulmschneider, P. 1990, in ASP Conf. Proc. 9, Cool Stars, Stellar Systems, and the Sun, ed. G. Wallerstein (San Francisco: ASP), 3

Ulmschneider, P., Priest, E. R., \& Rosner, R., ed. 1991, Mechanisms of Chromospheric and Coronal Heating (Berlin: Springer)

Ulmschneider, P., \& Stein, R. F. 1982, A\&A, 106, 9

Vilhu, O. 1987, in Cool Stars, Stellar Systems, and the Sun, ed. J. L. Linsky \& R. B. Stencel (Berlin: Springer), 110

Vilhu, O., et al. 1996, in Cool Stars, Stellar Systems, and the Sun, ed. R. Pallavicini \& A. K. Dupree, in press.

Vilhu, O., Tsuru, T., Cameron, A. C., Budding, E., Banks, T., Slee, B., Ehrenfreund, P., \& Foing, B. H. 1993, A\&A, 278, 467

Vogt, S. S., \& Penrod, G. D. 1983, PASP, 95, 565

Wood, B. E. 1996, Ph.D. thesis, Univ. of Colorado

Wood, B. E., Brown, A., Linsky, J. L., Kellett, B. J., Bromage, G. E., Hodgkin, S. T., \& Pye, J. P. 1994, ApJS, 93, 287

Wood, B. E., Harper, G. M., Linsky, J. L., \& Dempsey, R. C. 1996, ApJ, 458,761 
